\title{
Towards an enumerative geometry of the moduli space of twisted curves and $r$ th roots
}

\author{
Alessandro Chiodo
}

\begin{abstract}
The enumerative geometry of $r$ th roots of line bundles is crucial in the theory of $r$-spin curves and occurs in the calculation of Gromov-Witten invariants of orbifolds. It requires the definition of the suitable compact moduli stack and the generalization of the standard techniques from the theory of moduli of stable curves. In a previous paper, we constructed a compact moduli stack by describing the notion of stability in the context of twisted curves. In this paper, by working with stable twisted curves, we extend Mumford's formula for the Chern character of the Hodge bundle to the direct image of the universal $r$ th root in $K$-theory.
\end{abstract}

\section{Contents}

1 Introduction . . . . . . . . . . . . . . . . . . . . 1461

2 Previous results on moduli of curves and $r$ th roots . . . . . . . . . 1465

3 Grothendieck-Riemann-Roch theorem for the universal $r$ th root . 1473

4 Applications and motivations . . . . . . . . . . . . . . 1487

References .......................... . . 1494

\section{Introduction}

In this paper we investigate the enumerative geometry of the moduli of curves paired with the $r$ th roots of a given line bundle (usually the canonical bundle or the structure sheaf). It is well known that one can construct a proper and smooth stack $\overline{\mathcal{M}}_{g, n}^{r}$ of $r$ th roots of the canonical bundle of genus- $g$ curves. More generally, one can consider $r$ th roots of any power of the canonical bundle possibly twisted at the markings $x_{1}, x_{2}, \ldots, x_{n}$ with arbitrarily chosen multiplicities $m_{1}, m_{2}, \ldots, m_{n}$; see [Jar00], [AJ03], [CCC07], and [Chi08]. In Theorem 1.1.1, we determine the Chern character

$$
\operatorname{ch}\left(R^{\bullet} \pi_{*} \mathcal{S}\right),
$$

where $\pi: \mathcal{C} \rightarrow \overline{\mathcal{M}}_{g, n}^{r}$ is the universal curve and $\mathcal{S}$ is the universal $r$ th root. As illustrated in $\S 4$, the formula for $\operatorname{ch}\left(R^{\bullet} \pi_{*} \mathcal{S}\right)$ has direct applications to Gromov-Witten theory of stacks, to $r$-spin theory in the sense of Witten [Wit93] (see also Fan, Jarvis, and Ruan [FJR07] for recent generalizations and conjectures), and to the mathematics of Hurwitz numbers (see Zvonkine [Zvo08]).

Received 17 October 2006, accepted in final form 7 May 2008.

2000 Mathematics Subject Classification 14H10 (primary), 14H60 (secondary).

Keywords: enumerative geometry of curves, moduli stacks of curves, twisted curves.

Partially supported by the Marie Curie Intra-European Fellowship within the 6th European Community Framework Programme, MEIF-CT-2003-501940.

This journal is (C) Foundation Compositio Mathematica 2008. 


\section{A. Chiodo}

\subsection{Main theorem: Grothendieck-Riemann-Roch theorem for universal $r$ th roots}

Via the Grothendieck-Riemann-Roch (GRR) theorem, in Theorem 1.1.1, we express the Chern character of the perfect complex $R^{\bullet} \pi_{*} \mathcal{S}$ in terms of tautological classes: the kappa classes $\kappa_{d}$ and the psi classes $\psi_{1}, \ldots, \psi_{n}$. The calculation takes place on the stack $\overline{\mathcal{M}}_{g, n}^{r}$, the proper moduli space of $r$ th roots of the line bundle

$$
\mathcal{K}=\left(\omega^{\log }\right)^{\otimes s}\left(-\sum_{i=1}^{n} m_{i}\left[x_{i}\right]\right),
$$

where $s, m_{1}, \ldots, m_{n}$ are integers, and we adopt the notation

$$
\omega^{\log }=\omega\left(\sum_{i=1}^{n}\left[x_{i}\right]\right) .
$$

The kappa and psi classes

$$
\kappa_{d}=\pi_{*}\left(\mathrm{c}_{1}\left(\omega^{\log }\right)^{d+1}\right) \quad \text { and } \quad \psi_{i}=\mathrm{c}_{1}\left(\omega \mid \text { marking } x_{i}\right)
$$

are defined (see (12)) in the Chow ring of $\overline{\mathcal{M}}_{g, n}^{r}$ rather than in the Chow ring of $\overline{\mathcal{M}}_{g, n}$ as usually occurs. In view of applications to enumerative geometry, we provide a formula that allows us to compute intersection numbers directly via products in the tautological ring of $\overline{\mathcal{M}}_{g, n}$ (Corollary 3.1.8).

The case $r=1$ : Mumford's calculation for the Hodge bundle. The Hodge bundle $\mathbb{H}$ is the direct image of $\omega$ on $\overline{\mathcal{M}}_{g, n}$. In [Mum83], Mumford provides a formula for $\mathrm{ch}(\mathbb{H})$ in terms of tautological classes and Bernoulli numbers. In view of our generalization, it is convenient to reformulate Mumford's formula in terms of Bernoulli polynomials $B_{m}(x)$ by recalling the relation $B_{m}(1)=(-1)^{m} B_{m}$, where $B_{m}$ is the usual Bernoulli number. We rewrite Mumford's formula as

$$
\operatorname{ch}\left(R^{\bullet} \pi_{*} \omega\right)=\operatorname{ch}(\mathbb{H})-1=\sum_{d \geqslant 0}\left(\frac{B_{d+1}(1)}{(d+1) !} \kappa_{d}-\sum_{i=1}^{n} \frac{B_{d+1}(1)}{(d+1) !} \psi_{i}^{d}+\frac{1}{2} \frac{B_{d+1}(1)}{(d+1) !} j_{*}\left(\gamma_{d-1}\right)\right),
$$

where $j$ is the composite of $\pi$ and the natural double cover

$$
\text { Sing }^{\prime} \longrightarrow \text { Sing } \subset \mathcal{C} \text {. }
$$

(We recall that the singular locus Sing of $\mathcal{C} \rightarrow \overline{\mathcal{M}}_{g, n}$ consists of nodes and the category Sing' formed by nodes equipped with the choice of an order for the two branches of each node is a double cover.) The class $\gamma_{d} \in A^{d}\left(\mathrm{Sing}^{\prime}\right)$ is defined as follows. Let $\psi$ and $\widehat{\psi}$ be the first Chern classes of the line bundles whose fibre at each point $p^{\prime}$ of Sing' is the cotangent line along the first and the second branch, respectively. Then, we set

$$
\gamma_{d}:=\sum_{i+j=d}(-\psi)^{i} \widehat{\psi}^{j}
$$

and we let $\gamma_{d}$ be zero for $d<0$.

Mumford's equation [Mum83, (5.2)] for $\operatorname{ch}(\mathbb{H})$ is written in a different form, but the above expression can be obtained once the following remarks are made.

(i) Mumford does not use the standard notation for Bernoulli numbers recalled at Notation 3.1.2.

(ii) The definition of the kappa classes recalled above is the standard notation for marked curves and differs from the one of [Mum83], which does not involve the markings: in [Mum83] the kappa class is $\pi_{*}\left(\mathrm{c}_{1}(\omega)^{d+1}\right)$ rather than $\pi_{*}\left(\mathrm{c}_{1}\left(\omega^{\log }\right)^{d+1}\right)$. For example, Mumford's zeroth kappa class is $2 g-2$ rather than $\kappa_{0}=2 g-2+n$. In degree $d$ this change makes the term $-\sum_{i} \psi_{i}^{d}$ appear in our formula (see (13)).

(iii) Mumford has a typographical error in the index of the kappa term of his formula (the index ' $2 l+1$ ' should rather be ' $2 l-1$ '). 


\section{ENUMERATIVE GEOMETRY OF MODULI OF $r$ th ROOTS}

The case $r>1$ : stack-theoretic curves and Bernoulli polynomials. Our generalization of Mumford's theorem takes place on the proper moduli stack $\overline{\mathcal{M}}_{g, n}^{r}$ of $r$ th roots of

$$
\mathcal{K}=\left(\omega^{\log }\right)^{\otimes s}\left(-\sum_{i=1}^{n} m_{i}\left[x_{i}\right]\right),
$$

for $s, m_{1}, \ldots, m_{n} \in \mathbb{Z}$ and $(2 g-2+n) s-\sum_{i} m_{i} \in r \mathbb{Z}$. The formula involves Bernoulli polynomials evaluated at $s / r$ and $m_{i} / r$ (see Notation 3.1.2). In this way, Mumford's calculation corresponds to the case where $r, s$, and the indices $m_{i}$ are all equal to 1 and $\overline{\mathcal{M}}_{g, n}^{r}$ equals $\overline{\mathcal{M}}_{g, n}$.

In [Chi08], we show that the construction of $\overline{\mathcal{M}}_{g, n}^{r}$ is just a slight modification of the compactification $\overline{\mathcal{M}}_{g, n}$ of $\mathcal{M}_{g, n}$, which was obtained by Deligne and Mumford [DM69] by allowing stable curves (curves $C$ with nodes as singularities and finite automorphism group). The stack $\overline{\mathcal{M}}_{g, n}^{r}$ is also a compactification: it contains the stack $\mathcal{M}_{g, n}^{r}$ classifying smooth curves with an $r$ th root of $\mathcal{K}$. It is also obtained by enlarging the category $\mathcal{M}_{g, n}^{r}$ to new geometric objects: $r$ th roots of $\mathcal{K}$ on $r$-stable curves, which are stack-theoretic curves $\mathcal{C}$ with stable coarse space, representable smooth locus, and nodes $p: \operatorname{Spec} \mathbb{C} \rightarrow \mathcal{C}$ such that $\operatorname{Aut}_{\mathcal{C}}(p)$ is isomorphic to $\boldsymbol{\mu}_{r}$.

As in Mumford's calculation, we need to consider Sing, the stack classifying curves (equipped with an $r$ th root) alongside the choice of a singularity. As above, the stack Sing naturally maps to $\overline{\mathcal{M}}_{g, n}^{r}$ and has a natural double cover induced by the choice of a branch for each node; in this way we have the morphism

$$
j: \operatorname{Sing}^{\prime} \rightarrow \overline{\mathcal{M}}_{g, n}^{r} .
$$

The stack Sing' is naturally equipped with two line bundles whose fibres are the cotangent lines to the first branch of the coarse curve and the cotangent lines to the second branch. We write $\psi, \widehat{\psi} \in H^{2}\left(\mathrm{Sing}^{\prime}, \mathbb{Q}\right)$ for their respective first Chern classes. (In this notation, we privilege the coarse curve, because in this way the classes $\psi$ and $\widehat{\psi}$ are more easily related to the classes $\psi_{i}$.)

Recall that $\mathcal{C}$ is equipped with the universal $r$ th root $\mathcal{S}$. For each point $p^{\prime}$ of $\operatorname{Sing}^{\prime}$, the restriction of $\mathcal{S}$ to the first branch yields a multiplicity index $q\left(p^{\prime}\right) \in\{0, \ldots, r-1\}$ as follows. The line bundle $\mathcal{S}$ on the point lifting the node to the first branch can be regarded as a representation of $\boldsymbol{\mu}_{r}$. This representation is a power of the representation attached to the line cotangent to the first branch. We define $q\left(p^{\prime}\right)$ as the order of that power. By construction, such a multiplicity index is locally constant on Sing'. We get a decomposition of Sing' and the restriction morphisms

$$
\operatorname{Sing}^{\prime}=\bigsqcup_{q=0}^{r-1} \operatorname{Sing}_{q}^{\prime}, \quad j_{q}=\left.j\right|_{\operatorname{Sing}_{q}^{\prime}}: \operatorname{Sing}_{q}^{\prime} \rightarrow \overline{\mathcal{M}}_{g, n}^{r} \quad \text { for } 0 \leqslant q<r .
$$

Theorem 1.1.1. Let $s, m_{1}, \ldots, m_{n}$ be integers satisfying $(2 g-2+n) s-\sum_{i} m_{i} \in r \mathbb{Z}$. Let $\mathcal{S}$ be the universal $r$ th root of $\left(\omega^{\log }\right)^{\otimes s}\left(-\sum_{i=1}^{n} m_{i}\left[x_{i}\right]\right)$ on the universal $r$-stable curve $\mathcal{C}$ over the moduli stack $\overline{\mathcal{M}}_{g, n}^{r}$. The direct image $R^{\bullet} \pi_{*} \mathcal{S}$ via the universal curve $\pi: \mathcal{C} \rightarrow \overline{\mathcal{M}}_{g, n}^{r}$ satisfies the equation

$$
\operatorname{ch}\left(R^{\bullet} \pi_{*} \mathcal{S}\right)=\sum_{d \geqslant 0}\left(\frac{B_{d+1}(s / r)}{(d+1) !} \kappa_{d}-\sum_{i=1}^{n} \frac{B_{d+1}\left(m_{i} / r\right)}{(d+1) !} \psi_{i}^{d}+\frac{1}{2} \sum_{q=0}^{r-1} \frac{r B_{d+1}(q / r)}{(d+1) !}\left(j_{q}\right)_{*}\left(\gamma_{d-1}\right)\right),
$$

where the cycle $\gamma_{d}$ in $A^{d}\left(\operatorname{Sing}_{q}^{\prime}\right)$ is $\sum_{i+j=d}(-\psi)^{i} \widehat{\psi}^{j}$ (we set $\gamma_{d}=0$ when $d$ is negative).

The proof of Theorem 1.1.1 is given in $\S 3.1$.

\subsection{Applications}

Theorem 1.1.1 can be used to compute several invariants in the enumerative geometry of curves. In $\S 4$, we illustrate the relation with the crepant resolution conjecture in Gromov-Witten theory and with the theory of $r$-spin curves. 


\section{A. Chiodo}

The crepant resolution conjecture. The first context where Theorem 1.1.1 provides a useful application is the Gromov-Witten theory of orbifolds. Indeed, when we set $s=0$, Theorem 1.1.1 can be regarded as a tool for the computation of genus- $g$ Gromov-Witten invariants of the orbifold $\left[\mathbb{C}^{2} / \boldsymbol{\mu}_{r}\right]$, where $\boldsymbol{\mu}_{r}$ acts as $(x, y) \mapsto\left(\xi_{r} x, \xi_{r}^{-1} y\right)$. In $\S 4.1$, we detail the definition of such invariants, we spell out how Theorem 1.1.1 applies, and we provide some examples, which can be generalized for any value of $g$ and $r$. The crucial observation is that stable maps to $\left[\mathbb{C}^{2} / \boldsymbol{\mu}_{r}\right]$ are related to the $r$ th root of $\mathcal{O}(-D)$, where $D$ is a divisor supported on the markings.

The Gromov-Witten potential is the power series assembling all Gromov-Witten invariants. The potential of Gorenstein orbifolds such as $\left[\mathbb{C}^{2} / \boldsymbol{\mu}_{r}\right]$ is the subject of the crepant resolution conjecture. In the statement of Bryan and Graber [BG06], the conjecture predicts that the genus-0 potential of a Gorenstein orbifold and that of any crepant resolution of the coarse space are equal after a change of variables. Recently, this statement has been proved for the genus-0 case by Coates, Corti, Iritani and Tseng [CCIT07] and generalized to a conjecture involving higher genera by Coates and Ruan [CR07, Conjecture 10.2].

Invariants in $r$-spin theory. The theory of $r$-spin curves is the next natural context where Theorem 1.1.1 applies. By definition, $r$-spin structures are $r$ th roots of $\omega(-D)$, where $D$ is an effective divisor supported on the markings. This corresponds to the case $s=1$ (recent work by [FJR07] casts this notion in the wider framework of $W$-spin theory; we remark that this new notion directly involves higher values of $s$ ).

The crucial invariant of this theory is again a power series, the $r$-spin potential, assembling several intersection numbers in $\overline{\mathcal{M}}_{g, n}^{r}$ (see discussion in $\S 4.2$ ). This theory has been put in the framework of Gromov-Witten theory by Jarvis, Kimura, and Vaintrob [JKV01b]. In [FSZ06], Faber, Shadrin, and Zvonkine prove that the $r$-spin potential satisfies the Gelfand-Dikil hierarchy as conjectured by Witten in [Wit93] (the case $r=2$ is due to [Kon92]). In [CZ07], in collaboration with Zvonkine, Theorem 1.1.1 is used to provide a formula relating this Gromov-Witten $r$-spin potential to the twisted Gromov-Witten $r$-spin potential (the $r$-spin potentials extended with the Chern characters $\operatorname{ch}\left(R^{\bullet} \pi_{*} \mathcal{S}\right)$ ). Finally, Theorem 1.1 .1 provides new information on the enumerative geometry of $R^{\bullet} \pi_{*} \mathcal{S}$; we discuss in some examples the relation with the cycle $\mathrm{c}_{\mathrm{W}}$, the virtual fundamental cycle in Gromov-Witten $r$-spin theory.

We also point out that the Chern classes of $R^{\bullet} \pi_{*} \mathcal{S}$ are expected to be related to Hurwitz numbers via a conjecture by Zvonkine (see [Zvo08]), which generalizes the Ekedahl-Lando-ShapiroVainshtein (ELSV) formula (60) concerning the Chern classes of the Hodge bundle.

Grothendieck-Riemann-Roch formulae. The proof of Theorem 1.1.1 provides a new application of the GRR formula. In $\S 3$, we start from a stack-theoretic curve $\widetilde{C}$ equipped with the $r$ th root $\widetilde{S}$ on a base scheme $X$; then, we work in the cohomology ring of the desingularization $C$ of the coarse space $\bar{C}$ of $\widetilde{C}$. In this way, the curve $C$ is semistable and is equipped with a line bundle $S$ whose direct image on the base scheme $X$ coincides with that of the universal $r$ th root $\widetilde{S}$ on $\widetilde{C}$ (Lemma 2.2.5). This allows us to carry out the calculation in the scheme-theoretic context via the standard GRR formula for schemes.

The statement of Theorem 1.1.1 can also be obtained via Toen's GRR formula for stacks [Toe99]: this alternative approach is illustrated in $\S 3.3$ and the result coincides with the formula of Theorem 1.1.1. In this comparison, the main difficulty is the identification of the formula of Theorem 1.1.1, which lies in rational cohomology, with the formula given by Toen's theorem, which is naturally stated in terms of cohomology classes with 'coefficients in the representations'. We point out that this problem also occurs in the proof of Tseng's orbifold quantum Riemann-Roch [Tse05], the formula expressing the generating function of twisted Gromov-Witten invariants in terms of 


\section{ENUMERATIVE GEOMETRY OF MODULI OF $r$ th ROOTS}

the generating function of the untwisted invariants. There, stack-theoretic curves also occur, and Toen's GRR is applied; in [Tse05, $\S 7.2 .6$ ], by means of calculations in the $r$ th cyclotomic field, the 'coefficients in the representations' are expressed in terms of Bernoulli polynomials.

\subsection{Terminology}

Throughout this paper we work over the field of complex numbers $\mathbb{C}$.

Notation 1.3.1. By $r$ we always denote a positive integer. We often use the involution of $\{0, \ldots, r-1\}$ sending $q>0$ to $r-q$ and fixing 0 . We write it as $q \mapsto \overline{r-q}$, by adopting the convention $\bar{i}=i$ if $0 \leqslant i<r$ and $\bar{r}=0$.

Notation 1.3.2. We adopt the terminology of [LM00] for stacks. For Deligne-Mumford stacks there is a natural 2-functor to the category of algebraic spaces associating to a stack an algebraic space that is universal with respect to morphisms to algebraic spaces. We usually refer to it as the coarse space.

Notation 1.3.3. We often need to describe stacks and morphisms between stacks locally in terms of explicit equations. Let $X$ and $U$ be algebraic stacks and let $x \in X$ and $u \in U$ be geometric points. We say 'the local picture of $X$ at $x$ is given by $U$ (at $u$ )' if there is an isomorphism between the strict henselization $X^{\text {sh }}$ of $X$ at $x$ and the strict henselization $U^{\text {sh }}$ of $U$ at $u$.

Notation 1.3.4. Let $f: X \rightarrow Y$ be a proper birational morphism of complex varieties. We write $\operatorname{Ex}(f)$ for the exceptional locus in $X$ of the morphism $f$.

Notation 1.3.5 (Moduli of stable curves). For $2 g-2+n>0$, the category $\overline{\mathcal{M}}_{g, n}$ of stable curves of genus $g$ with $n$ markings is a proper, smooth, and irreducible stack of Deligne-Mumford type [DM69] of dimension $3 g-3+n$. The full subcategory $\mathcal{M}_{g, n}$ of smooth curves is an open substack.

Remark 1.3.6. We often consider nodal curves $C \rightarrow X$ with sections $s: X \rightarrow C$ in the smooth locus. We point out that there is a canonical isomorphism between $s^{*} \omega_{C / X}(s(X))$ and $\mathcal{O}_{X}$.

\subsection{Structure of the paper}

In $\S 2$ we recall the construction of $\overline{\mathcal{M}}_{g, n}^{r}$ and we set up the notation. In $\S 3$ we prove the theorem stated above. In $\S 4$, we illustrate the applications mentioned in $\S 1.2$.

\section{Previous results on moduli of curves and $r$ th roots}

In this section, we recall the definition of $\overline{\mathcal{M}}_{g, n}^{r}$, the proper moduli stack of $r$ th roots of

$$
\mathcal{K}=\left(\omega^{\log }\right)^{\otimes s}\left(-\sum_{i=1}^{n} m_{i}\left[x_{i}\right]\right),
$$

for $s, m_{1}, \ldots, m_{n} \in \mathbb{Z}$. For degree reasons, the category of $r$ th roots of $\mathcal{K}$ is nonempty if and only if we have

$$
s(2 g-2+n)-\sum_{i=1}^{n} m_{i} \in r \mathbb{Z} .
$$

It is known that there exists a proper stack of $r$ th roots of $\mathcal{K}$ (see [Jar00], [AJ03], [CCC07], and [Chi08]). Here we follow the construction of [Chi08], where we show that the only difficulty lies in defining a new compactification of $\mathcal{M}_{g, n}$. 


\section{A. Chiodo}

\subsection{Compactifying $\mathcal{M}_{g, n}$ using $r$-stable curves instead of stable curves}

Definition 2.1.1 ( $r$-stable curve). For $X$ a scheme and $C$ a stack, consider a proper and flat morphism $C \rightarrow X$ with $n$ ordered sections $x_{1}, \ldots, x_{n}: X \rightarrow C$, mapping into the smooth locus of $C \rightarrow X$, as below.

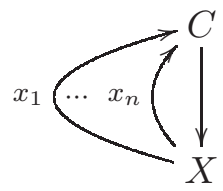

The data $\left(C \rightarrow X, x_{1}, \ldots, x_{n}\right)$ form an $r$-stable curve of genus $g$ with $n$ markings if:

(i) the fibres are purely one-dimensional with at most nodal singularities;

(ii) the smooth locus $C^{\text {sm }}$ is an algebraic space;

(iii) the coarse space $\bar{C} \rightarrow X$ with the sections $\bar{x}_{1}, \ldots, \bar{x}_{n}$ is a genus- $g n$-pointed stable curve $\left(\bar{C} \rightarrow X, \bar{x}_{1}, \ldots, \bar{x}_{n}\right)$; and

(iv) the local picture at a node is given by $\left[U / \boldsymbol{\mu}_{r}\right] \rightarrow T$, where

- $T=\operatorname{Spec} A$,

- $U=\operatorname{Spec} A[z, w] /(z w-t)$ for some $t \in A$, and

- the action of $\boldsymbol{\mu}_{r}$ is given by $(z, w) \mapsto\left(\xi_{r} z, \xi_{r}^{-1} w\right)$.

Remark 2.1.2. For any $r$-stable curve as above we denote by $\omega_{C / X}^{\log }$ the sheaf of logarithmic differentials defined as

$$
\omega_{C / X}^{\log }:=\omega_{C / X} \otimes \mathcal{O}\left(\sum_{i=1}^{n} x_{i}(X)\right) .
$$

If $\left(\bar{C} \rightarrow X, \bar{x}_{1}, \ldots, \bar{x}_{n}\right)$ is the corresponding coarse stable curve, we have [Chi08, Proposition 2.5.1]

$$
\pi_{C}^{*}\left(\omega_{\bar{C} / X}^{\log }\right) \cong \omega_{C / X}^{\log }
$$

For each $r$, the notion of $r$-stable curve yields a compactification of the stack of $n$-pointed genus- $g$ stable smooth curves (recall that stability in the case of smooth curves only means $2 g-2+n>0$ ). The following theorem is a consequence of a theorem of Olsson [Ols07] and is stated in this form in $[$ Chi08, 4.1.4, 4.2.7].

Theorem 2.1.3 [Ols07, Chi08]. The moduli functor $\overline{\mathcal{M}}_{g, n}(r)$ of $r$-stable $n$-pointed curves of genus $g$ forms a proper, smooth, and irreducible Deligne-Mumford stack of dimension $3 g-3+n$.

The stack of 1-stable curves $\overline{\mathcal{M}}_{g, n}(1)$ is the stack of stable curves $\overline{\mathcal{M}}_{g, n}$ in the sense of Deligne and Mumford. For any $r>1$ and for $2 g-2+n>0$, we get new compactifications of the stack of smooth n-pointed curves $\mathcal{M}_{g, n}$. There is a natural surjective, finite, and flat morphism $\overline{\mathcal{M}}_{g, n}(r) \rightarrow$ $\overline{\mathcal{M}}_{g, n}(1)$ that is invertible on the open dense substack of smooth $n$-pointed genus- $g$ curves and yields an isomorphism between coarse spaces. The morphism is not injective; indeed, the restriction of $\overline{\mathcal{M}}_{g, n}(r) \rightarrow \overline{\mathcal{M}}_{g, n}$ to the substack of singular $r$-stable curves has degree $1 / r$ as a morphism between stacks.

Remark 2.1.4 ( $\vec{l}$-stability). In fact, in [Chi08], we classify all compactifications of $\mathcal{M}_{g}$ inside Olsson's nonseparated stack of twisted curves: stack-theoretic curves with representable smooth locus and nodal singularities with finite stabilizers. In this way, we hit upon the notion of $\vec{l}$-stability, which we recall in passing even if it will not be used in this paper. For simplicity, let us consider the case of unmarked curves. A node of a stable curve over $\mathbb{C}$ has type 0 if the normalization of the stable curve at the node is connected, whereas it has type $i \in\{1, \ldots,\lfloor g / 2\rfloor\}$ if the normalization is the union of two disjoint curves of genus $i$ and $g-i$. For $\vec{l}=\left(l_{i} \mid i \in\{0,1, \ldots,\lfloor g / 2\rfloor\}\right)$, a twisted curve 


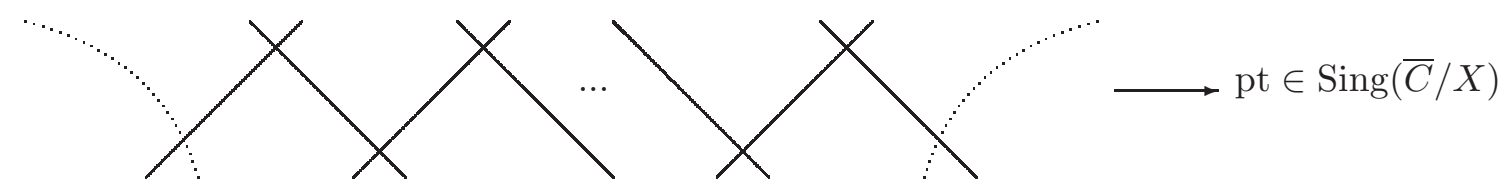

Figure 1. The fibre of $\operatorname{Ex}(C \rightarrow \bar{C}) \rightarrow \operatorname{Sing}(\bar{C} / X)$, a chain of $r-1$ rational lines.

is $\vec{l}$-stable if its nodes of type $i$ have stabilizers of order $l_{i}$. We point out that, when all entries of $\vec{l}$ equal $r$, an $\vec{l}$-stable curve is nothing more than an $r$-stable curve in the sense of Definition 2.1.1.

Remark 2.1.5 (singular locus of universal $r$-stable curve). In $\S 3$, we work in the étale topology of $\overline{\mathcal{M}}_{g, n}(r)$. Let $X$ be a scheme and $X \rightarrow \overline{\mathcal{M}}_{g, n}(r)$ be an étale morphism; then, the corresponding $r$-stable $X$-curve is a morphism $\widetilde{\pi}: \widetilde{C} \rightarrow X$ whose variation is maximal: the Kodaira-Spencer homomorphism

$$
T \overline{\mathcal{M}}_{g, n}(r)_{x} \rightarrow \operatorname{Ext}^{1}\left(\mathcal{C}_{x}, \mathcal{O}_{\mathcal{C}_{x}}\right)
$$

is surjective for any geometric point $x$ in $\overline{\mathcal{M}}_{g, n}(r)$. In this case, the following facts derive from the theory of moduli of nodal curves and $r$-stable curves (see [Ols07] and [Chi08]).

(A) The stack $\widetilde{C}$. The $r$-stable curve $\widetilde{C}$ is a regular stack and the singular locus $\operatorname{Sing}(\widetilde{C} / X)$ of $\widetilde{\pi}: \widetilde{C} \rightarrow X$ is regular of codimension 2 in $\widetilde{C}$. The image of $\operatorname{Sing}(\widetilde{C} / X)$ in $X$ with respect to $\pi$ is a normal crossing divisor (the pullback in $X$ of the boundary locus via $X \rightarrow \overline{\mathcal{M}}_{g, n}(r)$; see [Ols07]). Note that the local picture of $\widetilde{\pi}$ at any point $x \in \operatorname{Sing}(\widetilde{C} / X)$ is given by

$$
\left[\operatorname{Spec} U / \boldsymbol{\mu}_{r}\right] \rightarrow \operatorname{Spec} T
$$

where $T$ equals $\mathbb{C}\left[x_{1}, \ldots, x_{3 g-3+n}\right], U$ equals $T[z, w] /\left(z w=x_{1}\right)$, and the group $\boldsymbol{\mu}_{r}$ acts as $(z, w) \mapsto\left(\xi_{r} z, \xi_{r}^{-1} w\right)$.

(B) The coarse space $\bar{C}$ of $\widetilde{C}$. If we consider the coarse space of $\widetilde{C}$, then we get an algebraic scheme $\bar{C}$ with singularities of type $A_{r-1}$ on every point of the singular locus. More precisely, using the local picture given at the previous point, we see that the singular locus $\operatorname{Sing}(\bar{C} / X)$ of the stable curve $\bar{\pi}: \bar{C} \rightarrow X$ is a regular scheme, has codimension 2 in $\bar{C}$, and the local picture of $\bar{\pi}$ at any of its points is given by the spectrum of

$$
\mathbb{C}\left[x_{1}, \ldots, x_{3 g-3+n}\right] \longrightarrow \mathbb{C}\left[x_{1}, \ldots, x_{3 g-3+n}, z, w\right] /\left(z w=x_{1}^{r}\right) .
$$

Note that $\widetilde{\pi}(\operatorname{Sing}(\widetilde{C} / X))$ equals $\bar{\pi}(\operatorname{Sing}(\bar{C} / X))$ and is a normal crossings divisor in $X$.

(C) The desingularization $C$ of $\bar{C}$. By repeating $\lfloor r / 2\rfloor$ blowups of the singular locus starting from $\bar{C}$ we get the desingularization $\pi: C \rightarrow \bar{C}$ of $\bar{\pi}: \bar{C} \rightarrow X$. The morphism $\pi: C \rightarrow X$ is a semistable curve. The fibre of $\pi$ is sketched in Figure 1 (the exceptional locus of $C \rightarrow \bar{C}$ is drawn with continuous lines and the rest of the curve with dotted lines). The scheme $C$ is regular, and the singular locus $\operatorname{Sing}(C / X)$ is a regular scheme, of codimension 2 inside $C$, and in fact it is an étale cover of $\operatorname{Sing}(\bar{C} / X)$ of degree $r$. Note that, for $r>1$, $\operatorname{Sing}(C / X)$ lies in the exceptional locus $\operatorname{Ex}(C \rightarrow \bar{C})$ of the desingularization $\nu: C \rightarrow \bar{C}$ (this happens because for $r>1$ every node of the curve $\bar{C} \rightarrow X$ is a singular point of $\bar{C}$ ).

We now show how the compactification $\overline{\mathcal{M}}_{g, n}(r)$ illustrated above allows us to define easily a proper stack of $r$ th roots of $\mathcal{K}$. The above description of the singular loci will be generalized and further developed in Remark 2.2.3. 


\section{A. Chiodo}

\subsection{Roots of order $r$ on $r$-stable curves}

Once the moduli stack of $r$-stable curves is defined, the construction follows naturally. We fix $s, m_{1}, \ldots, m_{n} \in \mathbb{Z}$ and we assume that $(2 g-2+n) s-\sum_{i} m_{i}$ is a multiple of $r$. The category of $r$ th roots of

$$
\mathcal{K}=\left(\omega^{\log }\right)^{\otimes s}\left(-\sum_{i} m_{i}\left[x_{i}\right]\right)
$$

on stable curves with $n$ markings $x_{i} \in C$ forms a stack that is not proper; instead, we consider $r$ th roots of $\mathcal{K}$ on $r$-stable curves.

Theorem 2.2.1 [Chi08, Theorem 4.2.3]. The moduli functor $\overline{\mathcal{M}}_{g, n}^{r}$ of $r$ th roots of $\mathcal{K}$ on $r$-stable curves forms a proper and smooth stack of Deligne-Mumford type of dimension $3 g-3+n$.

For $s=m_{1}=\cdots=m_{n}=0, \mathcal{K}$ is $\mathcal{O}$ and the stack $\overline{\mathcal{M}}_{g, n}^{r}$ is a group stack $\mathcal{G}$ on $\overline{\mathcal{M}}_{g, n}(r)$. In general $\overline{\mathcal{M}}_{g, n}^{r}$ is a finite torsor on $\overline{\mathcal{M}}_{g, n}(r)$ under $\mathcal{G}$.

The morphism $\overline{\mathcal{M}}_{g, n}^{r} \rightarrow \overline{\mathcal{M}}_{g, n}(r)$ is étale. It factors through a morphism locally isomorphic to $B \boldsymbol{\mu}_{r} \rightarrow$ Spec $\mathbb{C}$ (a $\boldsymbol{\mu}_{r}$-gerbe) and a representable étale $r^{2 g}$-fold cover; therefore it is has degree $r^{2 g-1}$.

Remark 2.2.2. In fact, in [Chi08, Theorem 4.2.3] we completely determine the values of $\vec{l}$ for which we have the properness of the stack of $r$ th roots of $\mathcal{K}$ on curves satisfying $\vec{l}$-stability in the sense illustrated in Remark 2.1.4. As explained above, when all entries of the multi-index $\vec{l}$ equal $r$, saying that a curve is $r$-stable is equivalent to saying that a curve is $\vec{l}$-stable. From the point of view of enumerative geometry, all choices of $\vec{l}$ are equivalent, because the rational cohomology of a DeligneMumford stack only depends on the coarse space, and the coarse space of the compactification does not depend on $\vec{l}$.

Remark 2.2.3 (analysis of singular locus). As we did for the stack of $r$-stable curves $\overline{\mathcal{M}}_{g, n}(r)$, we study an object of $\overline{\mathcal{M}}_{g, n}^{r}$ over a base scheme $X$ mapping to $\overline{\mathcal{M}}_{g, n}^{r}$ via an étale morphism. Via pull back we get an $r$-stable curve $\widetilde{\pi}: \widetilde{C} \rightarrow X$ and an $r$ th $\operatorname{root} \widetilde{S}$ of $\left(\omega_{C / X}^{\log }\right)^{\otimes s}\left(-\sum_{i} m_{i}\left[x_{i}\right]\right)$. These data involve several local geometric structures, for which we fix notation for the rest of the paper.

(a) We write

$$
\widetilde{Z}=\operatorname{Sing}(\widetilde{C} / X) \hookrightarrow \widetilde{C}
$$

for the singular locus of the $r$-stable curve $\widetilde{\pi}: \widetilde{C} \rightarrow X$. Let

$$
\widetilde{\varepsilon}: \widetilde{Z}^{\prime} \rightarrow \widetilde{Z}
$$

be the 2-fold étale cover classifying the two possible ways of ordering the incident branches. We denote by

$$
\widetilde{\iota}: \widetilde{Z}^{\prime} \rightarrow \widetilde{Z}^{\prime}
$$

the natural involution switching the order. In this way, if we pull back $\widetilde{C} \rightarrow X$ via $\widetilde{j}: \widetilde{Z}^{\prime} \rightarrow X$,

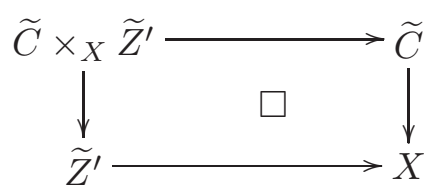

we get a curve $\widetilde{C} \times_{X} \widetilde{Z}^{\prime} \longrightarrow \widetilde{Z}^{\prime}$ such that over each point of $\widetilde{Z}^{\prime}$ there is a prescribed choice of a node and of a branch of that node. We use this fact in order to show that the stack $Z^{\prime}$ admits a natural decomposition

$$
\widetilde{Z}^{\prime}=\bigsqcup_{q=0}^{r-1} \widetilde{Z}_{q}^{\prime}
$$




\section{ENUMERATIVE GEOMETRY OF MODULI OF $r$ th ROOTS}

To this effect, we define a multiplicity index $q: \widetilde{Z}^{\prime} \rightarrow\{0, \ldots, r-1\}$ and we realize $\widetilde{Z}_{0}^{\prime}, \ldots, \widetilde{Z}_{r-1}^{\prime}$ as the loci where this index is constant. Consider a geometric point of $p^{\prime} \in \widetilde{Z}^{\prime}$ and the corresponding node in the $r$-stable curve $\widetilde{C} \times_{X} \widetilde{Z}^{\prime} \longrightarrow \widetilde{Z}^{\prime}$. The line cotangent to the prescribed branch is a line bundle on the node: a faithful $\boldsymbol{\mu}_{r}$-representation. The restriction of the $r$ th root $\widetilde{S}$ to the node can also be regarded as a $\boldsymbol{\mu}_{r}$-representation: a power of order $q \in\{0, \ldots, r-1\}$ of the cotangent line to the prescribed branch. In this way, the locally constant function $q$ is defined, and so are $\widetilde{Z}_{0}^{\prime}, \ldots, \widetilde{Z}_{r-1}^{\prime}$.

(b) Let $\bar{\pi}: \bar{C} \rightarrow X$ be the coarse space of $\widetilde{\pi}: \widetilde{C} \rightarrow X$. We write

$$
Z=\operatorname{Sing}(\bar{C} / X) \hookrightarrow \bar{C}
$$

for the singular locus of the stable curve $\bar{C} \rightarrow X$. As above, set

$$
\bar{\varepsilon}: Z^{\prime} \rightarrow Z \quad \text { and } \quad \bar{\imath}: Z^{\prime} \rightarrow Z^{\prime}
$$

for the natural 2-fold étale cover of $Z$ and the involution of $Z^{\prime}$. By

$$
\bar{j}: Z^{\prime} \rightarrow X
$$

we denote the composite of $Z \rightarrow \bar{C}$ and $\bar{C} \rightarrow X$. We write

$$
\tau=\mathrm{c}_{1}\left(\mathcal{N}_{\bar{j}}^{\vee}\right) \in A^{1}\left(Z^{\prime}\right)
$$

for the first Chern class of the conormal sheaf $\mathcal{N}_{\bar{j}}^{\vee}$ of $\bar{j}: Z^{\prime} \rightarrow X$.

(c) The scheme $Z^{\prime}$ is the coarse space of the stack $\widetilde{Z}^{\prime}$. The natural decomposition of (a) induces a decomposition

$$
Z^{\prime}=\bigsqcup_{q=0}^{r-1} Z_{q}^{\prime}
$$

We write

$$
\bar{j}_{q}: Z_{q}^{\prime} \rightarrow X
$$

for the restriction of $\bar{j}: Z^{\prime} \rightarrow X$ to $Z_{q}^{\prime}$. We write $\tau_{q}$ for the restriction of $\tau$ to $Z_{q}$ (when there is no ambiguity we simply write $\tau$ ).

(d) Let $\widetilde{L}$ be the line bundle over $\widetilde{Z}^{\prime}$ whose fibre over a point of $p^{\prime} \in \widetilde{Z}^{\prime}$ is the line cotangent to the prescribed branch associated to $p^{\prime}$. The cotangent lines to the remaining branch yield the line bundle $\widetilde{\iota}^{*} \widetilde{L}$. By $\bar{L}$ and $\tau^{*} \bar{L}$, we denote the analogue line bundles on $Z^{\prime}$. Since $Z^{\prime}$ is the coarse space of $\widetilde{Z}^{\prime}$, we can pull $L$ back to $Z^{\prime}$ via $p: \widetilde{Z}^{\prime} \rightarrow Z^{\prime}$; we get

$$
p^{*} \bar{L}=\widetilde{L}^{\otimes r} .
$$

We write

$$
\psi=\mathrm{c}_{1}(\bar{L}), \quad \widehat{\psi}=\mathrm{c}_{1}\left(\bar{\iota}^{*} \bar{L}\right) \quad \text { in } A^{1}\left(Z_{q}^{\prime}\right) .
$$

Clearly, we have $\widehat{\psi}=\iota^{*} \psi$.

(e) Consider the above desingularization $\nu: C \rightarrow \bar{C}$ (see Figure 1). The exceptional locus $\operatorname{Ex}(\nu) \subset$ $C$ is a curve over $Z \subset \bar{C}$ whose fibre is a chain of $r-1$ rational lines. It can be regarded as a 


\section{A. Chiodo}

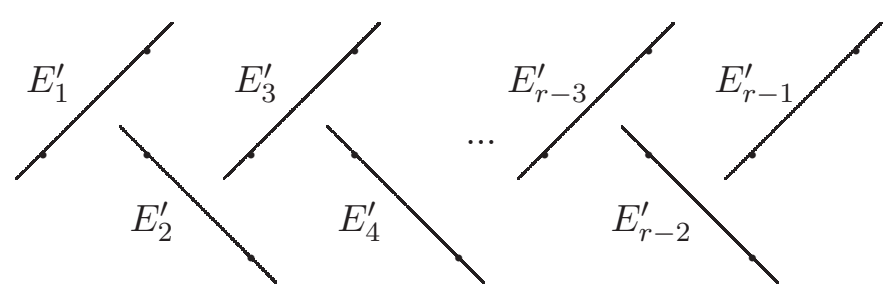

normalization

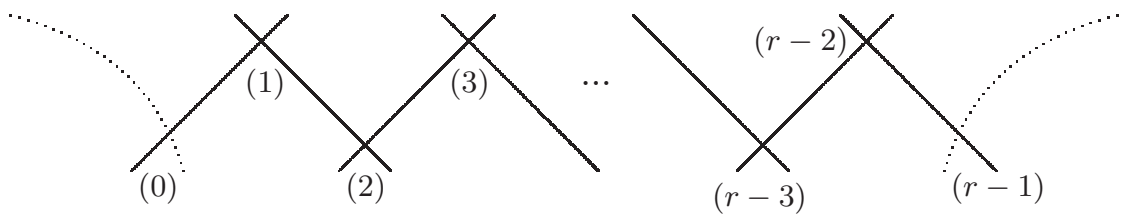

FiguRE 2. The normalization of the fibre over $Z^{\prime}$.

subcurve of $C \times_{X} Z \rightarrow Z$. We pull back these $Z$-curves to $Z^{\prime}$ via $\bar{\varepsilon}: Z^{\prime} \rightarrow Z$.

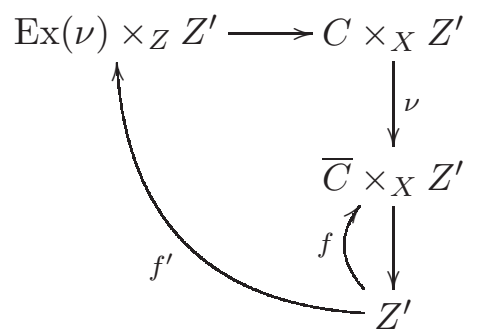

The curve $\bar{C} \times_{X} Z^{\prime} \rightarrow Z^{\prime}$ has a distinguished section $f: Z^{\prime} \rightarrow \bar{C} \times_{X} Z^{\prime}$ in the singular locus and, by the definition of $Z^{\prime}$, all along this section there is a distinguished choice of a branch meeting $f\left(Z^{\prime}\right)$. This induces a section $f^{\prime}$ mapping $Z^{\prime}$ to the singular locus of $C \times_{X} Z^{\prime}$ so that for any $p^{\prime} \in Z^{\prime}$ the node $f^{\prime}\left(p^{\prime}\right)$ lifts $f\left(p^{\prime}\right)$ and has a branch lying over the chosen branch of $f\left(p^{\prime}\right)$. In the above curve $\operatorname{Ex}(\nu) \times{ }_{Z} Z^{\prime} \longrightarrow Z^{\prime}$ each fibre is a chain of $r-1$ rational lines, which can now be numbered with indices $i$ running from 1 to $r-1$ starting from the node $f^{\prime}\left(Z^{\prime}\right)$. We write

$$
E_{i}^{\prime} \rightarrow Z^{\prime}
$$

for the $\mathbb{P}^{1}$-bundles on $Z^{\prime}$ obtained from the normalization of $\operatorname{Ex}(\nu) \times{ }_{Z} Z^{\prime} \longrightarrow Z^{\prime}$ (see Figure 2). Pulling back $E_{i}^{\prime} \rightarrow Z^{\prime}$ via $Z_{q}^{\prime} \hookrightarrow Z$ we finally get

$$
E_{q, i}^{\prime} \rightarrow Z_{q}^{\prime}
$$

(f) Note that the fibre of the exceptional locus $\operatorname{Ex}(\nu) \times_{Z} Z^{\prime}$ over $Z^{\prime}$ contains nodes of $C \times{ }_{X} Z^{\prime}$, which are naturally labelled from 0 to $r-1$ starting from $f^{\prime}\left(Z^{\prime}\right)$. In this way the singular locus of $C \times_{X} Z^{\prime} \rightarrow Z^{\prime}$ can be regarded as the disjoint union of $r$ copies $\left(Z^{\prime}\right)^{(i)}$ of $Z^{\prime}$ for $i \in\{0, \ldots, r-1\}$. Over $Z_{q}^{\prime}$ we have $r$ copies $\left(Z_{q}^{\prime}\right)^{(0)}, \ldots,\left(Z_{q}^{\prime}\right)^{(r-1)}$. We write

$$
\lambda_{q, i}: Z_{q}^{\prime} \rightarrow E_{q, i}^{\prime} \quad(\text { for } i \neq 0) \quad \text { and } \quad \widehat{\lambda}_{q, i}: Z_{q}^{\prime} \rightarrow E_{q, i+1}^{\prime} \quad(\text { for } i \neq r-1)
$$

for the sections lifting the $i$ th node $\left(Z_{q}^{\prime}\right)^{(i)}$ to the bundles $E_{q, i}^{\prime}$ and $E_{q, i+1}^{\prime}$ (see Figure 2). We write $L_{q, i}$ and $\widehat{L}_{q, i}$ for the corresponding conormal sheaves. By setting $L_{q, 0}=\bar{L}$ and $\widehat{L}_{q, r-1}=\bar{\iota}^{*} \bar{L}$ 


\section{ENUMERATIVE GEOMETRY OF MODULI OF $r$ th ROOTS}

we extend the definition of $L_{q, i}$ and $\widehat{L}_{q, i}$ to all $0 \leqslant q, i \leqslant r-1$. We set

$$
\psi_{q, i}=\mathrm{c}_{1}\left(L_{q, i}\right), \quad \widehat{\psi}_{q, i}=\mathrm{c}_{1}\left(\widehat{L}_{q, i}\right) \quad \text { in } A^{1}\left(Z_{q}^{\prime}\right), \quad \text { for } 0 \leqslant q, i \leqslant r-1 .
$$

(g) Note that the involution $\iota$ interchanging the two sheets of $Z^{\prime}$ yields a natural involution of $\operatorname{Ex}(\nu) \times{ }_{Z} Z^{\prime} \longrightarrow Z^{\prime}$ and of its normalization. The involution maps $Z_{q}^{\prime}$ to $Z_{\overline{r-q}}^{\prime}$ and the $i$ th component of the curve $\operatorname{Ex}(\nu) \times_{Z} Z^{\prime} \longrightarrow Z^{\prime}$ to the $(r-i)$ th component. In this way, the above $\mathbb{P}^{1}$-bundle $E_{q, i}^{\prime} \rightarrow Z_{q}^{\prime}$ is sent to the $\mathbb{P}^{1}$-bundle $E_{\overline{r-q}, r-i}^{\prime} \rightarrow Z_{r-q}^{\prime}$. These two $\mathbb{P}^{1}$-bundles are sent via the projection to $C$ to the same divisor, which we denote by

$$
E_{q, i} \in \operatorname{Div}(C) .
$$

Clearly $E_{q, i}$ and $E_{\overline{r-q}, r-i}$ denote the same divisor in $C$. The exceptional locus of the desingularization $C \rightarrow \bar{C}$ can be written as

$$
\operatorname{Ex}(C \rightarrow \bar{C})=\sum_{q=0}^{\lfloor r / 2\rfloor} H_{q},
$$

where

$$
H_{q}= \begin{cases}\sum_{1 \leqslant i \leqslant r-1} E_{q, i} & \text { for } 0<q<r / 2, \\ \sum_{1 \leqslant i \leqslant\lfloor r / 2\rfloor} E_{q, i} & \text { if } q=0 \text { or } q=r / 2 .\end{cases}
$$

(h) We write

$$
\sigma_{q, i}: Z_{q}^{\prime} \rightarrow E_{q, i} \quad \text { and } \quad \widehat{\sigma}_{q, i}: Z_{q}^{\prime} \rightarrow E_{q, i+1}
$$

for the composition of $\lambda_{q, i}$ and $\widehat{\lambda}_{q, i}$ of (4) with the normalization. We write

$$
\rho_{q, i}: E_{q, i} \hookrightarrow C
$$

for the natural inclusion of $E_{q, i}$ in $C$. Note that $\rho_{q, i} \circ \sigma_{q, i}$ and $\rho_{q, i} \circ \widehat{\sigma}_{q, i}$ coincide; we denote such a composite morphism by

$$
j_{q, i}: Z_{q}^{\prime} \rightarrow C .
$$

The morphisms above fit in the following commutative diagram.

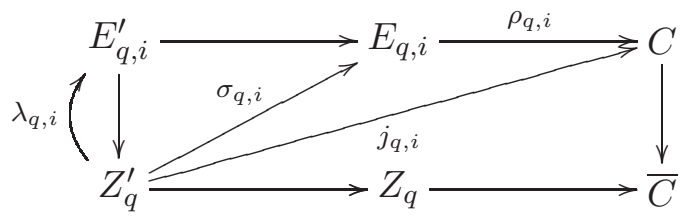

(The diagram remains commutative if we replace $\lambda_{q, i}, \sigma_{q, i}$, and $j_{q, i}$ with $\widehat{\lambda}_{q, i-1}, \widehat{\sigma}_{q, i-1}$, and $j_{q, i-1 .)}$

(i) The characteristic classes $\psi_{q, i}, \widehat{\psi}_{q, i}, \tau$ satisfy the following relations:

$$
\begin{aligned}
\widehat{\psi}_{q, i} & =-\psi_{q, i+1}, \\
\tau & =\psi_{q, i}+\widehat{\psi}_{q, i} .
\end{aligned}
$$

The first relation follows from the fact that $\psi_{q, i}$ and $\psi_{q, i+1}$ are the first Chern classes of two conormal line bundles corresponding to two disjoint sections of the $\mathbb{P}^{1}$-bundle $E_{q, i+1}^{\prime} \rightarrow Z_{q}^{\prime}$. The two line bundles are dual to each other; so, their respective first Chern classes are opposite. The second relation also involves first Chern classes. On the left hand side we have $c_{1}$ of the conormal line bundle $\mathcal{N}_{\bar{j}}^{\vee}$ with respect to $\bar{j}: Z^{\prime} \rightarrow X$. On the right-hand side we have $c_{1}$ of 


\section{A. Chiodo}
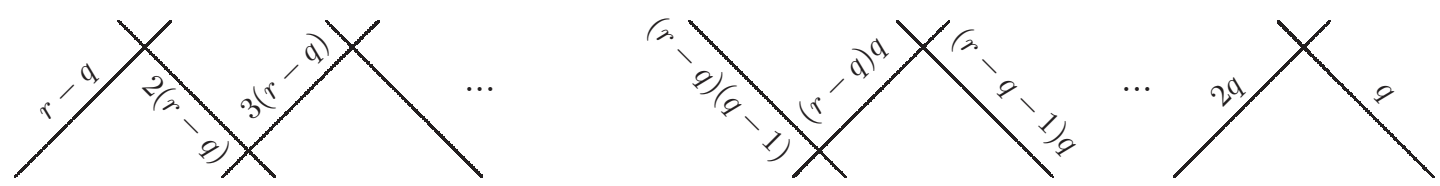

FiguRE 3. The $q$-chain divisor.

$\left(\operatorname{det} \mathcal{N}_{f^{\prime}}\right)^{\vee}$, where $f^{\prime}$ is the morphism $Z^{\prime} \rightarrow C^{\prime}=C \times_{X} Z^{\prime}$. The two line bundles are isomorphic; indeed, by the definition of the normal sheaf, we have

$$
\begin{aligned}
\operatorname{det} \mathcal{N}_{f^{\prime}} & \cong\left(\operatorname{det} T_{Z^{\prime}}\right)^{\vee} \otimes f^{*} \operatorname{det} T_{C^{\prime}} \cong \mathcal{N}_{\bar{j}} \otimes\left(\bar{j}^{*} \operatorname{det} T_{X}\right)^{\vee} \otimes f^{*} \operatorname{det} T_{C^{\prime}} \\
& \cong \mathcal{N}_{\bar{j}} \otimes f^{*}\left(\operatorname{det} T_{C^{\prime}} \otimes\left(\pi^{*} \operatorname{det} T_{X}\right)^{\vee}\right) \cong \mathcal{N}_{\bar{j}} \otimes f^{*} \omega_{\widetilde{C} / X} \cong \mathcal{N}_{\bar{j}},
\end{aligned}
$$

where the last equality holds because $f^{*} \omega_{\widetilde{C} / X}$ is trivial (recall Remark 1.3.6 and the definition of the relative cotangent sheaf of a nodal curve; the claim extends to $r$-stable curves by (3)). As an immediate consequence of the above equations, we have the relation $\psi_{q, i+1}=\psi_{q, i}-\tau$, for all $i=0, \ldots, r-1$. Therefore, using $\psi_{q, 0}=c_{1}(\bar{L})=\psi$, we get

$$
\psi_{q, i}=\psi-i \tau \text {. }
$$

Furthermore, summing up the above equations (6) for $i=0, \ldots, r-1$, we get $\tau=(\psi+\widehat{\psi}) / r$. Hence we have

$$
\psi_{q, i}=\frac{(r-i) \psi-i \widehat{\psi}}{r}
$$

We conclude this section with a result allowing us to handle the Grothendieck-Riemann-Roch calculation. We define the $q$-chain divisor $D_{q}$ needed in the statement. We illustrate the definition with a picture (Figure 3). Let us fix $q \in] 1, r / 2\left[\right.$ for simplicity; then we define $D_{q}$ by summing the divisors $E_{q, i}$ for $i$ ranging from $r-1$ to 1 with multiplicities ranging from $q$ to $r-q$ as follows. We start with $E_{q, r-1}$ with multiplicity $q$ and we increase the multiplicity with step $q$ up to $(r-q) q E_{q, q}$; then, we decrease with step $r-q$ until we reach $(r-q) E_{q, 1}$.

We give the formal definition of $D_{q}$ :

$$
D_{q}= \begin{cases}\sum_{1 \leqslant i \leqslant q}(r-q) i E_{q, i}+\sum_{q<i<r} q(r-i) E_{q, i} & \text { for } 0<q<r \text { and } q \neq r / 2, \\ \sum_{1 \leqslant i \leqslant q}(r-q) i E_{q, i}=\sum_{q<i<r} q(r-i) E_{q, i} & \text { for } q=r / 2 \text { and } q=0 .\end{cases}
$$

Clearly $D_{0}$ vanishes by definition, whereas $D_{q}=D_{r-q}$ for $0<q<r$ and $q \neq r / 2$.

Example 2.2.4. Let us consider a nodal curve given by the union of two curves $C^{\prime}$ and $C^{\prime \prime}$ meeting at one node. Assume that the node is replaced by a chain $H$ of $r-1$ projective lines as above with the first component $E_{q, 1}$ attached to $C^{\prime}$ and the last component $E_{q, r-1}$ attached to $C^{\prime \prime}$. Then, the $q$-chain divisor supported on $H$ has degree $-r$ on the component $E_{q, q}, 0$ on the remaining components of the chain, $r-q$ on $C^{\prime}$, and $q$ on $C^{\prime \prime}$.

Lemma 2.2.5. In the situation described above, let $\widetilde{S}$ be the $r$ th root on $\widetilde{C}$, let $\bar{S}$ be its direct image on $\bar{C}$, and let $\nu: C \rightarrow \bar{C}$ be the desingularization. There exists a line bundle $S$ on $C$ satisfying

$$
S^{\otimes r} \cong \nu^{*} \mathcal{K} \otimes \mathcal{O}\left(-\sum_{1 \leqslant q \leqslant\lfloor r / 2\rfloor} D_{q}\right)
$$




\section{ENUMERATIVE GEOMETRY OF MODULI OF $r$ th ROOTS}

and

$$
\nu_{*} S=\bar{S}
$$

Proof. The statement follows from results of Cornalba [Cor89] for square roots generalized to the case $r>2$ by Jarvis [Jar00, Jar01] and by Caporaso, Casagrande, and Cornalba [CCC07]. We contract the divisors,

$$
E_{q, i} \quad \text { for } 0 \leqslant q \leqslant\lfloor r / 2\rfloor \text { and } i \neq q
$$

(in particular all divisors $E_{0, i}$ are contracted). In this way, we obtain a semistable curve $Q \rightarrow X$ and a morphism $\nu_{Q}: Q \rightarrow \bar{C}$, whose exceptional locus is a divisor fibred in projective lines; more precisely, by construction, $\operatorname{Ex}\left(\nu_{Q}\right)$ is the disjoint union of the images $I_{q}$ of

$$
E_{q, q} \text { for } 0<q \leqslant\lfloor r / 2\rfloor
$$

via $C \rightarrow Q$. As soon as $r>2, I_{q}$ is not a Cartier divisor: each fibre of $I_{q}$ on $\bar{C}$ contains two singular points, whose local pictures in $Q$ are given by $\left\{x y=t^{q}\right\}$ and $\left\{x y=t^{r-q}\right\}$ in $\mathbb{C}^{\operatorname{dim} X+2}$. On the other hand the divisor $q(r-q) I_{q}$ is a Cartier divisor in $Q$ and, if we regard $I_{q}$ as a $\mathbb{P}^{1}$-bundle, on each fibre $F \cong \mathbb{P}^{1} \subseteq I_{q}$ on $\bar{C}$ we have

$$
\left.\operatorname{deg}\left(q(r-q) I_{q}\right)\right|_{F}=-\frac{q(r-q)}{q}-\frac{q(r-q)}{r-q}=-r .
$$

Jarvis [Jar01, Theorem 3.3.9] shows that there is a line bundle $S_{Q}$ on the semistable curve $Q$ that is sent to $\bar{S}$ via direct image to $\bar{C}$, and is equipped with an isomorphism

$$
S_{Q}^{\otimes r} \stackrel{\sim}{\longrightarrow} \nu_{Q}^{*} \mathcal{K} \otimes \mathcal{O}\left(-\sum_{q} q(r-q) I_{q}\right),
$$

(see [Jar01], in particular points 1-3 after Lemma 3.3.8, the proof of Theorem 3.3.9, and Figure 1 therein). Indeed, $Q$ is realized as $\operatorname{Proj}\left(\bigoplus_{k \geqslant 0} \operatorname{Sym}^{k}(\bar{S})\right)$ and, with respect to such an isomorphism, $S_{Q}$ is identified with $\mathcal{O}(1)$; see [CCC07, $\S 4.2$ and Proposition 4.2.2] and Falting's description of torsion-free sheaves in [Fal96] and [Jar98, $\S \S 3.1$ and 3.2]. In fact, it should also be noted that the realization of the direct image of $S_{Q}$ as a direct image from the stack $\widetilde{C}$ is due to Abramovich and Jarvis [AJ03, Proposition 4.3.1].

It is easy to see that pulling $q(r-q) I_{q}$ back to $C$ via $C \rightarrow Q$ yields the divisor $D_{q}$; therefore, the pullback of $S_{Q}$ to $C$ yields the line bundle $S$ satisfying the properties stated above.

\section{Grothendieck-Riemann-Roch theorem for the universal $r$ th root}

We work with the moduli stack $\overline{\mathcal{M}}_{g, n}^{r}$ of $r$ th roots of $\mathcal{K}$ on $r$-stable curves. Let $\mathcal{C}$ be the universal $r$-stable curve, and let $\mathcal{S}$ be the universal $r$ th root.

\subsection{The proof of the main theorem}

Notation 3.1 .1 (tautological classes). For any $r$-stable $n$-pointed curve $\widetilde{C} \rightarrow X$ with markings $x_{1}, \ldots, x_{n}: X \rightarrow C$, we write

$$
\kappa_{d}:=\pi_{*}\left(\mathrm{c}_{1}\left(\omega_{\widetilde{C} / X}^{\log }\right)^{d+1}\right) \quad \text { and } \quad \psi_{i}=\mathrm{c}_{1}\left(x_{i}^{*} \omega_{\widetilde{C} / X}\right) .
$$

By [AC87], we have

$$
\kappa_{d}=\pi_{*}\left(\mathrm{c}_{1}\left(\omega_{\widetilde{C} / X}\right)^{d+1}\right)+\sum_{i=1}^{n} \psi_{i}^{d} .
$$

The cohomology classes and relations introduced above are usually given in the literature for the category of stable curves $\overline{\mathcal{M}}_{g, n}$; however, they equally hold for $r$-stable curves by (3). 


\section{A. Chiodo}

Notation 3.1.2 (Bernoulli polynomials and numbers). The Bernoulli polynomials $B_{n}(x)$ are defined by

$$
\frac{e^{t x} t}{e^{t}-1}=\sum_{n=0}^{\infty} \frac{B_{n}(x)}{n !} t^{n}
$$

We recall that the Bernoulli polynomials satisfy the property $B_{n}(x)=(-1)^{n} B_{n}(1-x)$ and

$$
B_{n}(x+y)=\sum_{m=0}^{n}\left(\begin{array}{l}
n \\
m
\end{array}\right) B_{m}(x) y^{n-m}
$$

which can be regarded as a formula expressing Bernoulli polynomials in terms of Bernoulli numbers $B_{n}(0)$.

Proof of Theorem 1.1.1. We prove the theorem in the étale topology of the stack of $r$ th roots of $\mathcal{K}$; therefore, we work directly with $\widetilde{C}, \bar{C}$, and $C$ over a base scheme $X$, and the $r$ th root $\widetilde{S}$ on $\widetilde{C}$. In order to calculate $\operatorname{ch}\left(R^{\bullet} \widetilde{\pi}_{*} \widetilde{S}\right)$ we proceed in three steps as follows.

(1) We consider the desingularization $\pi: C \rightarrow X$ of $\bar{C} \rightarrow X$. By Lemma 2.2.5, $R^{\bullet} \widetilde{\pi}_{*} \widetilde{S}$ equals $R^{\bullet} \pi_{*} S$, for a line bundle $S \in \operatorname{Pic}(C)$ whose first Chern class can be expressed in the rational Chow ring in terms of $K=\mathrm{c}_{1}\left(\omega_{C / X}\right)$, the divisors $\Delta_{1}, \ldots, \Delta_{n}$ specifying the markings, and the $q$-chain divisors $D_{q}$. The calculation boils down to two distinct terms on which we focus in the second and third steps of the proof.

(2) We focus on the terms involving $K$ and $\Delta_{i}$.

(3) We conclude the calculation on the boundary locus.

Step 1. The curve $C \rightarrow X$ is a flat and proper morphism between regular schemes. Therefore, we can apply the Grothendieck-Riemann-Roch (GRR) theorem in the form

$$
\operatorname{ch}\left(R^{\bullet} \pi_{*} S\right)=\pi_{*}\left(\operatorname{ch}(S) \operatorname{td}^{\vee}\left(\Omega_{C / X}^{1}\right)\right) .
$$

We adopt Mumford's notation for the Todd character: for any line bundle $R$ we have

$$
\operatorname{td}^{\vee}(R)=\frac{\mathrm{c}_{1}(R)}{\exp \left(\mathrm{c}_{1}(R)\right)-1}
$$

Recall the exact sequence $0 \rightarrow \Omega_{C / X}^{1} \rightarrow \omega_{C / X} \rightarrow \omega_{C / X} \otimes \mathcal{O}_{Z} \rightarrow 0$ and write

$$
\operatorname{ch}\left(R^{\bullet} \pi_{*} S\right)=\pi_{*}\left((\operatorname{ch} S)\left(\operatorname{td}^{\vee} \omega_{C / X}\right)\left(\operatorname{td}^{\vee} \mathcal{O}_{\operatorname{Sing}(C / X)}\right)^{-1}\right) .
$$

By (10), the right-hand side equals

$$
\pi_{*}\left(\exp \left(s K / r-\sum_{i=1}^{n}\left(m_{i}-s\right) \Delta_{i} / r-\sum_{q=1}^{\lfloor r / 2\rfloor} D_{q} / r\right) \frac{K}{\exp (K)-1}\left(\operatorname{td}^{\vee} \mathcal{O}_{\operatorname{Sing}(C / X)}\right)^{-1}\right),
$$

where $K$ is $c_{1}\left(\omega_{C / X}\right)$. We make the following observations.

(i) For each $i$ the divisor $\Delta_{i}$ is disjoint from $\operatorname{Sing}(C / X)$.

(ii) The intersections between $K$ and any cycle in $\operatorname{Sing}(C / X)$ vanish (see Remark 1.3.6).

(iii) The intersections between $K$ and any divisor $D_{q}$ vanish (in other words $C \rightarrow \bar{C}$ is crepant).

(iv) The divisor $D_{q}$ is disjoint from $D_{q^{\prime}}$ for distinct indices $q$ and $q^{\prime}$ satisfying $0 \leqslant q, q^{\prime} \leqslant\lfloor r / 2\rfloor$.

In this way, the term (16) is the direct image via $\pi_{*}$ of

$$
\left(\frac{K \exp (s K / r)}{\exp (K)-1} \exp \left(-\sum_{i=1}^{n}\left(m_{i}-s\right) \Delta_{i} / r\right)\right)+\left[\left(\exp \left(-\sum_{q=1}^{\lfloor r / 2\rfloor} D_{q} / r\right)\left(\operatorname{td}^{\vee} \mathcal{O}_{\operatorname{Sing}(C / X)}\right)^{-1}\right)-1\right] \text {. }
$$




\section{ENUMERATIVE GEOMETRY OF MODULI OF $r$ th ROOTS}

Steps 2 and 3 are devoted to the first and the second summand of (17), respectively.

Step 2. We consider the term

$$
\pi_{*}\left(\frac{K \exp (s K / r)}{\exp (K)-1} \exp \left(-\sum_{i=1}^{n}\left(m_{i}-s\right) \Delta_{i} / r\right)\right) .
$$

Using Bernoulli polynomials we expand the term inside the brackets in the formula above as the product of

$$
\sum_{d \geqslant-1} \frac{B_{d+1}(s / r)}{(d+1) !} K^{d+1} \text { and } \prod_{i=1}^{n} \sum_{l \geqslant 0} \frac{\left(m_{i}-s\right)^{l}\left(-\Delta_{i}\right)^{l}}{l ! r^{l}} .
$$

The direct image via $\pi$ of terms of degree 0 vanishes and the divisors $\Delta_{i}$ are disjoint; therefore, we are only interested in the direct image of the sum

$$
\sum_{d \geqslant 0} \frac{B_{d+1}(s / r)}{(d+1) !} K^{d+1}+\sum_{i=1}^{n} \sum_{h, l \geqslant 0} \frac{\left(\left(m_{i}-s\right) / r\right)^{l+1} B_{h}(s / r)\left(-\Delta_{i}\right)^{l+1} K^{h}}{(l+1) ! h !} .
$$

By Remark 1.3.6, for all $i=1, \ldots, n$ we have $-K \Delta_{i}=\Delta_{i}^{2}$. Therefore, $\left(-\Delta_{i}\right)^{l+1} K^{h}$ equals $-K^{h+l} \Delta_{i}$ and we rewrite the above sum as

$$
\sum_{d \geqslant 0} \frac{B_{d+1}(s / r)}{(d+1) !} K^{d+1}-\sum_{i=1}^{n} \sum_{h, l \geqslant 0} \frac{\left(\left(m_{i}-s\right) / r\right)^{l+1} B_{h}(s / r) \Delta_{i} K^{h+l}}{(l+1) ! h !} .
$$

We now identify the term of degree $d+1$ in the above sum (since $\pi$ is of relative dimension 1 the terms of degree $d+1$ contribute to the degree- $d$ term of $\mathrm{ch}$ ). The first summand contains only one term of degree $d+1$ : the class $B_{d+1}(s / r) K^{d+1} /(d+1)$ !. The contribution of the second summand in degree $d$ occurs for each $i=1, \ldots, n$ and equals $K^{d} \Delta_{i}$ times

$$
-\sum_{h=0}^{d} \frac{\left(\left(m_{i}-s\right) / r\right)^{d-h+1} B_{h}(s / r)}{(d-h+1) ! h !}=-\frac{1}{(d+1) !} \sum_{h=0}^{d+1}\left(\begin{array}{c}
d+1 \\
h
\end{array}\right) B_{h}(s / r)\left(\frac{m_{i}-s}{r}\right)^{d+1-h}+B_{d+1}(s / r) .
$$

Using (15) we get

$$
\sum_{h=0}^{d+1}\left(\begin{array}{c}
d+1 \\
h
\end{array}\right) B_{h}(s / r)\left(\frac{m_{i}-s}{r}\right)^{d+1-h}=B_{h}\left(\frac{m_{i}-s}{r}+\frac{s}{r}\right)=B_{h}\left(\frac{m_{i}}{r}\right)
$$

and we simplify the above term:

$$
\left(-\frac{B_{d+1}\left(m_{i} / r\right)}{(d+1) !}+\frac{B_{d+1}(s / r)}{(d+1) !}\right) K^{d} \Delta_{i} .
$$

By $(13), \kappa_{d}=\pi_{*}\left(K^{d+1}\right)+\sum_{i=1}^{n} \psi_{i}^{d}$. Recall that $\psi_{i}^{d}=\pi_{*}\left(K^{d} \Delta_{i}\right)$. Putting everything together we express the term (18) as

$$
\frac{B_{d+1}(s / r)}{(d+1) !} \kappa_{d}-\sum_{i=1}^{n} \frac{B_{d+1}\left(m_{i} / r\right)}{(d+1) !} \psi_{i}^{d}
$$

Step 3. We now consider the second summand in (17). We omit $\pi_{*}(1)$ because $\pi$ is a morphism of relative dimension 1 . Therefore, we focus on the degree- $d$ term of

$$
\pi_{*}\left(\exp \left(-\sum_{1 \leqslant q \leqslant\lfloor r / 2\rfloor} D_{q} / r\right)\left(\operatorname{td}^{\vee} \mathcal{O}_{\operatorname{Sing}(C / X)}\right)^{-1}\right)
$$




\section{A. Chiodo}

and show that it equals

$$
\frac{1}{2} \sum_{q=0}^{r-1} \frac{r B_{d+1}(q / r)}{(d+1) !}\left(\bar{j}_{q}\right)_{*}\left(\gamma_{d-1}\right)
$$

By Remark 2.2.3(f) we regard the singular locus of $C \times_{X} Z^{\prime}$ as the union of copies $\left(Z_{q}^{\prime}\right)^{(i)}$ of $Z_{q}^{\prime}$ for $i=$ $0, \ldots, r-1$. Then, we can rewrite Mumford's formula [Mum83, Lemma 5.1] for $\left(\operatorname{td}^{\vee} \mathcal{O}_{\operatorname{Sing}(C / X)}\right)^{-1}$ as

$$
\left(\operatorname{td}^{\vee} \mathcal{O}_{\operatorname{Sing}(C / X)}\right)^{-1}=1+\frac{1}{2} \sum_{m \geqslant 1} \sum_{0 \leqslant q, i \leqslant r-1} \frac{B_{m+1}(0)}{(m+1) !}\left(j_{q, i}\right)_{*}\left(\frac{\psi_{q, i}^{m}+\widehat{\psi}_{q, i}^{m}}{\psi_{q, i}+\widehat{\psi}_{q, i}}\right)
$$

(see Remark 2.2.3(h) for the definition of $j_{q, i}$ ). Then, we replace $\left(\operatorname{td}^{\vee} \mathcal{O}_{\operatorname{Sing}(C / X)}\right)^{-1}$ in (20) to give

$$
\pi_{*}\left(\left(\prod_{q=1}^{\lfloor r / 2\rfloor} \sum_{h \geqslant 0} \frac{\left(-D_{q} / r\right)^{h}}{h !}\right)\left(1+\frac{1}{2} \sum_{0 \leqslant q, i<r} \sum_{m \geqslant 1} \frac{B_{m+1}(0)}{(m+1) !}\left(j_{q, i}\right)_{*}\left(\frac{\psi_{q, i}^{m}+\widehat{\psi}_{q, i}^{m}}{\psi_{q, i}+\widehat{\psi}_{q, i}}\right)\right)\right)
$$

and we expand using the fact that the divisors $D_{1}, \ldots, D_{\lfloor r / 2\rfloor}$ are disjoint,

$$
\pi_{*}\left(\left(1+\sum_{q=1}^{\lfloor r / 2\rfloor} \sum_{h \geqslant 0} \frac{\left(-D_{q} / r\right)^{h+1}}{(h+1) !}\right)\left(1+\frac{1}{2} \sum_{0 \leqslant q, i<r} \sum_{m \geqslant 1} \frac{B_{m+1}(0)}{(m+1) !}\left(j_{q, i}\right)_{*}\left(\frac{\psi_{q, i}^{m}+\widehat{\psi}_{q, i}^{m}}{\psi_{q, i}+\widehat{\psi}_{q, i}}\right)\right)\right) .
$$

We adopt the convention

$$
\widehat{q}=\overline{r-q} \text { for all } q \in\{0, \ldots, r-1\} .
$$

In this way, we have the following lemma.

Lemma 3.1.3. For $0 \leqslant q, i \leqslant r$ we have

$$
\left(j_{q, i}\right)^{*}\left(-D_{q}\right)= \begin{cases}\widehat{q} \psi & \text { for } i<q, \\ q \widehat{\psi} & \text { for } i \geqslant q .\end{cases}
$$

Proof. We use the notation established in Remark 2.2.3(h) and we calculate $\left(j_{q, i}\right)^{*} E_{q, i}$ by factoring $j_{q, i}$ as the composite of $\rho_{q, i}$ and $\sigma_{q, i}$. Similarly, we calculate $\left(j_{q, i}\right)^{*} E_{q, i+1}$ by factoring $j_{q, i}$ as the composite of $\rho_{q, i+1}$ and $\widehat{\sigma}_{q, i}$. We get

$$
\left(j_{q, i}\right)^{*} E_{q, i}=\left(\sigma_{q, i}\right)^{*} \rho_{q, i}^{*} E_{q, i}=\left(\sigma_{q, i}\right)^{*} \mathrm{c}_{1}\left(\mathcal{N}_{E_{q, i} / C}\right)
$$

and, similarly, $\left(j_{q, i}\right)^{*} E_{q, i+1}=\left(\widehat{\sigma}_{q, i}\right)^{*} c_{1}\left(\mathcal{N}_{E_{q, i+1} / C}\right)$. Therefore, $\left(j_{q, i}\right)^{*} E_{q, i}$ is the first Chern class of the line bundle whose fibres are tangent along the branches orthogonal to $E_{q, i}$. In this way, we have

$$
\left(j_{q, i}\right)^{*} E_{q, i}=-\widehat{\psi}_{q, i} \text { and }\left(j_{q, i}\right)^{*} E_{q, i+1}=-\psi_{q, i} .
$$

This implies the desired relations. Consider the case $1 \leqslant q<r / 2$. We apply the definition of $D_{q}$ and the relations of Remark 2.2.3(f); we get

$$
\begin{aligned}
-\left(j_{q, i}\right)^{*} D_{q} & =\sum_{i=1}^{q}(r-q) i\left(j_{q, i}\right)^{*}\left(-E_{q, i}\right)+\sum_{i=q+1}^{r-1} q(r-i)\left(j_{q, i}\right)^{*}\left(-E_{q, i}\right) \\
& = \begin{cases}(r-q)\left(i \widehat{\psi}_{q, i}+(i+1) \psi_{q, i}\right) & \text { if } i \leqslant q-1, \\
q\left((r-i) \widehat{\psi}_{q, i}+(r-i-1) \psi_{q, i}\right) & \text { if } i \geqslant q,\end{cases} \\
& = \begin{cases}(r-q)\left(i \tau+\psi_{q, i}\right) & \text { if } i \leqslant q-1, \\
q\left((r-i) \tau-\psi_{q, i}\right) & \text { if } i \geqslant q,\end{cases} \\
& = \begin{cases}\left.(r-q) \psi\right|_{Z_{q}^{\prime}} & \text { if } i \leqslant q-1, \\
\left.q(r \tau-\psi)\right|_{Z_{q}^{\prime}} & \text { if } i \geqslant q .\end{cases}
\end{aligned}
$$

This completes the proof of Lemma 3.1.3. 


\section{ENUMERATIVE GEOMETRY OF MODULI OF $r$ th ROOTS}

We return to the proof of the theorem. We expand the formula obtained in (23) to get

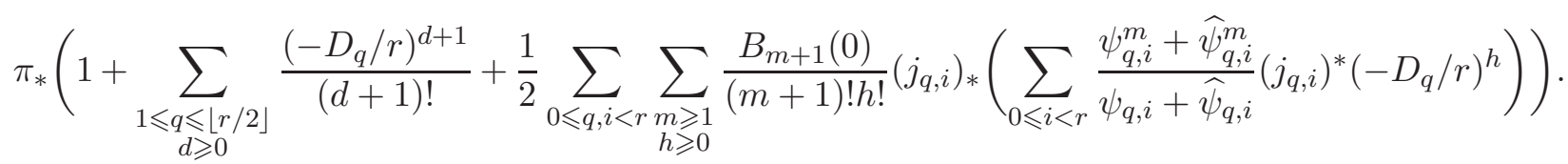

We split the summation over $i$ into a first summation ranging over the indices $i<q$ and a second summation ranging over the indices $i \geqslant q$. In this way, we can apply Lemma 3.1.3 and the above equation can be rewritten as follows (we omit $\pi_{*}(1)$ because it vanishes and we write $\pi \circ j_{q, i}$ as $\bar{j}_{q}$ )

$$
\begin{aligned}
& \pi_{*}\left(\sum_{\substack{1 \leqslant q \leqslant\lfloor r / 2\rfloor \\
d \geqslant 0}} \frac{\left(-D_{q} / r\right)^{d+1}}{(d+1) !}\right) \\
& \quad+\frac{1}{2(d+1) !} \sum_{0 \leqslant q, i<r}\left(\bar{j}_{q}\right)_{*}\left(\sum_{m=1}^{d} B_{m+1}(0)\left(\begin{array}{c}
d+1 \\
m+1
\end{array}\right) \sum_{i=0}^{q-1} \frac{\psi_{q, i}^{m}+\widehat{\psi}_{q, i}^{m}}{\psi_{q, i}+\widehat{\psi}_{q, i}}(\widehat{q} \psi / r)^{d-m}\right. \\
& \left.\quad+\sum_{m=1}^{d} B_{m+1}(0)\left(\begin{array}{c}
d+1 \\
m+1
\end{array}\right) \sum_{i=q}^{r-1} \frac{\psi_{q, i}^{m}+\widehat{\psi}_{q, i}^{m}}{\psi_{q, i}+\widehat{\psi}_{q, i}}(q \widehat{\psi} / r)^{d-m}\right) .
\end{aligned}
$$

Note that, when $m$ is odd, $\widehat{\psi}_{q, i}^{m}$ is opposite to $\psi_{q, i+1}^{m}$ and recall that $\psi_{q, i}+\widehat{\psi}_{q, i}$ equals $\tau=\psi / r+\widehat{\psi} / r$. Therefore the sum of the terms $\psi_{q, i}^{m}+\widehat{\psi}_{q, i}^{m}$ over the indices $m \in\{0, \ldots, q-1\}$ yields $\psi_{q, 0}^{m}+\widehat{\psi}_{q, q-1}^{m}$, hence $\psi_{q, 0}^{m}-\psi_{q, q}^{m}$. In this way, using the relation $\psi_{q, q}=\widehat{q} \psi / r-q \widehat{\psi} / r$ from (8), we rewrite the term between brackets in the second summand above as the sum $\alpha_{d-1, q}+\widehat{\alpha}_{d-1, q}$, where $\alpha_{d, q}$ is defined as

$$
\alpha_{d, q}=\frac{\sum_{m=0}^{d} B_{m+2}(0)\left(\begin{array}{c}
d+2 \\
m+2
\end{array}\right)\left((r \psi)^{m+1}-(\widehat{q} \psi-q \widehat{\psi})^{m+1}\right)(\widehat{q} \psi)^{d-m}}{r^{d}(\psi+\widehat{\psi})}
$$

and $\widehat{\alpha}_{d, q}$ is defined as $\alpha_{d, q}$ after the exchanges $\psi \leftrightarrow \widehat{\psi}$ and $q \leftrightarrow \widehat{q}$. Finally, we simplify the expression for $\alpha_{d, q}$ using $a^{m+1}-b^{m+1}=(a-b) \sum_{i=1}^{m} a^{i} b^{m-i}$ and $(r \psi)-(\widehat{q} \psi-q \widehat{\psi})=q \psi+q \widehat{\psi}$ :

$$
\alpha_{d, q}=\sum_{m=0}^{d} \frac{B_{m+2}(0)}{r^{d}}\left(\begin{array}{c}
d+2 \\
m+2
\end{array}\right) q(\widehat{q} \psi)^{d-m} \sum_{i+j=m}(r \psi)^{i}(\widehat{q} \psi-q \widehat{\psi})^{j} .
$$

Lemma 3.1.4. For $d \geqslant 1$, we have

$$
\sum_{q=1}^{\lfloor r / 2\rfloor} \pi_{*}\left(\left(-D_{q} / r\right)^{d+1}\right)=-\frac{1}{2} \sum_{q=0}^{r-1}\left(\bar{j}_{q}\right)_{*}\left(\frac{q \widehat{q}}{r^{d}} \sum_{a+b=d-1}(\widehat{q} \psi)^{a}(q \widehat{\psi})^{b}\right) .
$$

Proof. The proof uses the morphisms $\rho_{q, i}, \sigma_{q, i}$, and $\widehat{\sigma}_{q, i-1}$ introduced in Remark 2.2.3(h). We recall the commutative diagram

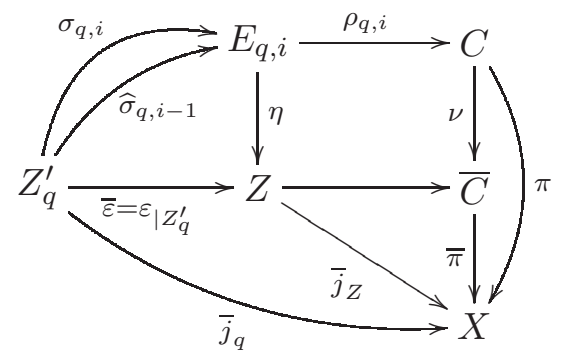

where $\eta$ denotes the projection of the $\mathbb{P}^{1}$-bundle $E_{q, i}$ to $Z$ and $\bar{j}_{Z}$ is the restriction of $\bar{\pi}$ to $Z \subset \bar{C}$. 


\section{A. Chiodo}

We write the cycle $E_{q, i}$ in $C$ as

$$
E_{q, i}=\pi^{*}\left(\bar{j}_{Z}\right)_{*}(1)-\sum_{j \neq i} E_{q, j}
$$

and we calculate the restriction of $E_{q, i}$ to itself:

$$
\begin{aligned}
\left(\rho_{q, i}\right)^{*} E_{q, i} & =\left(\rho_{q, i}\right)^{*} \pi^{*}\left(\bar{j}_{Z}\right)_{*}(1)-\left(\rho_{q, i}\right)^{*} \sum_{j \neq i} E_{q, j} \\
& =\eta^{*}\left(\bar{j}_{Z}\right)^{*}\left(\bar{j}_{Z}\right)_{*}(1)-\left(\rho_{q, i}\right)^{*} \sum_{j \neq i} E_{q, j} \\
& =-\eta^{*} \tau_{Z}- \begin{cases}\left(\widehat{\sigma}_{q, i-1}\right)_{*}(1) & q=i=r / 2, \\
\left(\widehat{\sigma}_{q, i-1}\right)_{*}(1)+\left(\sigma_{q, i}\right)_{*}(1) & \text { otherwise },\end{cases}
\end{aligned}
$$

where $\tau_{Z}=-\left(\bar{j}_{Z}\right)^{*}\left(\bar{j}_{Z}\right)_{*}(1)$ is the first Chern class of the conormal bundle relative to $\bar{j}_{Z}: Z \rightarrow X$. We now consider the pullback of $D_{q}$; let $1 \leqslant q \leqslant r / 2$; the definition of $D_{q}$ yields

$$
\left(\rho_{q, i}\right)^{*} D_{q}= \begin{cases}(r-q)\left(-i \eta^{*} \tau_{Z}-\left(\widehat{\sigma}_{q, i-1}\right)_{*}(1)+\left(\sigma_{q, i}\right)_{*}(1)\right) & 1 \leqslant i<q, \\ -(r-q) q \eta^{*} \tau_{Z}-(r-q)\left(\widehat{\sigma}_{q, q-1}\right)_{*}(1)-q\left(\sigma_{q, q}\right)_{*}(1) & i=q \neq r / 2, \\ -(r-q) q \eta^{*} \tau_{Z}-(r-q)\left(\widehat{\sigma}_{q, q-1}\right)_{*}(1) & i=q=r / 2, \\ q\left(-(r-i) \eta^{*} \tau_{Z}+\left(\widehat{\sigma}_{q, i-1}\right)_{*}(1)-\left(\sigma_{q, i}\right)_{*}(1)\right) & q<i<r .\end{cases}
$$

For $1 \leqslant q<r / 2$, we have

$$
\left(-D_{q}\right)^{d+1}=-\sum_{i=1}^{q}(r-q) i\left(\rho_{q, i}\right)_{*}\left(\left(\rho_{q, i}\right)^{*}\left(-D_{q}\right)^{d}\right)-\sum_{i=q+1}^{r-1} q(r-i)\left(\rho_{q, i}\right)_{*}\left(\left(\rho_{q, i}\right)^{*}\left(-D_{q}\right)^{d}\right),
$$

which can be written explicitly as

$$
\begin{aligned}
& -\sum_{i=1}^{q-1}(r-q)^{d+1} i\left(\rho_{q, i}\right)_{*}\left(\left(\widehat{\sigma}_{q, i-1}\right)_{*}(1)-\left(\sigma_{q, i}\right)_{*}(1)+i \eta^{*} \tau_{Z}\right)^{d} \\
& \quad-(r-q) q\left(\rho_{q, q}\right)_{*}\left((r-q)\left(\widehat{\sigma}_{q, q-1}\right)_{*}(1)+q\left(\sigma_{q, q}\right)_{*}(1)+(r-q) q \eta^{*} \tau_{Z}\right)^{d} \\
& \quad-\sum_{i=q+1}^{r-1} q^{d+1}(r-i)\left(\rho_{q, i}\right)_{*}\left(\left(\sigma_{q, i}\right)_{*}(1)-\left(\widehat{\sigma}_{q, i-1}\right)_{*}(1)+(r-i) \eta^{*} \tau_{Z}\right)^{d}
\end{aligned}
$$

(for $q=r / 2$, the above formula holds if we erase the third line and the term $q\left(\sigma_{q, q}\right)_{*}(1)$ in the second line). We finally expand each summand in the equation above. Recall that, if $h$ is positive and $a, b$, and $c$ commute and satisfy $b c=0$, we have

$$
(a+b+c)^{d}=a^{d}+\sum_{m=0}^{d-1}\left(\begin{array}{c}
d \\
m
\end{array}\right) a^{m}\left(b^{d-m}+c^{d-m}\right) .
$$

For $t>0$, we have the relations

$$
\left(\left(\widehat{\sigma}_{q, i-1}\right)_{*}(1)\right)^{t}=\left(\widehat{\sigma}_{q, i-1}\right)_{*}\left(\left(-\widehat{\psi}_{q, i-1}\right)^{t-1}\right) \quad \text { and } \quad\left(-\left(\sigma_{q, i}\right)_{*}(1)\right)^{t}=-\left(\sigma_{q, i}\right)_{*}\left(\left(\psi_{q, i}\right)^{t-1}\right) .
$$

So, for any $t>0$ and $l \geqslant 0$, we have

$$
\left(\eta^{*} \tau_{Z}\right)^{l}\left(-\left(\sigma_{q, i}\right)_{*}(1)\right)^{t}=-\left(\sigma_{q, i}\right)_{*}\left(\left(\psi_{q, i}\right)^{t-1}\left(\sigma_{q, i}\right)^{*}\left(\eta^{*} \tau_{Z}\right)^{l}\right)=-\left(\sigma_{q, i}\right)_{*}\left(\left(\psi_{q, i}\right)^{t-1} \tau^{l}\right)
$$

and similarly for $\widehat{\sigma}_{q, i-1}$. We are interested in the direct image of $\left(-D_{q}\right)^{d+1}$ via $\pi$; we remark that $\pi_{*} \eta^{*} \tau_{Z}=\left(\bar{j}_{Z}\right)_{*} \eta_{*} \eta^{*} \tau_{Z}$ vanishes (as a consequence of $\eta_{*} \eta^{*}=0$ ). We also note that both $\pi_{*}\left(\rho_{q, q}\right)_{*}\left(\widehat{\sigma}_{q, i-1}\right)_{*}$ and $\pi_{*}\left(\rho_{q, q}\right)_{*}\left(\widehat{\sigma}_{q, i}\right)_{*}$ equal $\left(\bar{j}_{q}\right)_{*}$. Finally, we recall the relation $\psi_{q, i}=-\widehat{\psi}_{q, i-1}$ 


\section{ENUMERATIVE GEOMETRY OF MODULI OF $r$ th ROOTS}

from (5), and we deduce that the direct image via $\pi$ of all summands in the above formulae (28) corresponding to $i \neq q$ vanishes. (The key fact in this is that $\widehat{\psi}_{q, i-1}+\psi_{q, i}=0$, which implies that

$$
\left(\bar{j}_{q}\right)_{*}\left((i \tau)^{m}\left(\left(-\widehat{\psi}_{q, i-1}\right)^{d-1-m}-\psi_{q, i}^{d-1-m}\right)\right)=0
$$

for $0 \leqslant m \leqslant d-1$.)

The direct image $\pi_{*}\left(\left(-D_{q}\right)^{d+1}\right)$ boils down to

$$
\begin{cases}-(r-q) q \pi_{*}\left(\rho_{q, q}\right)_{*}\left((r-q)\left(\widehat{\sigma}_{q, q-1}\right)_{*}(1)+q\left(\sigma_{q, q}\right)_{*}(1)+(r-q) q \tau\right)^{d} & q<r / 2 \\ -(r-q) q \pi_{*}\left(\rho_{q, q}\right)_{*}\left((r-q)\left(\widehat{\sigma}_{q, q-1}\right)_{*}(1)+(r-q) q \tau\right)^{d} & q=r / 2\end{cases}
$$

Then (29) yields

$$
\pi_{*}\left(\left(-D_{q}\right)^{d+1}\right)= \begin{cases}-(r-q)^{d+1} q \sum_{m=0}^{d-1}\left(\begin{array}{c}
d \\
m
\end{array}\right)\left(\bar{j}_{q}\right)_{*}\left(\left(-\widehat{\psi}_{q, q-1}\right)^{d-1-m}(q \tau)^{m}\right) & \\
-q^{d+1}(r-q) \sum_{m=0}^{d-1}\left(\begin{array}{c}
d \\
m
\end{array}\right)\left(\bar{j}_{q}\right)_{*}\left(\left(-\psi_{q, q}\right)^{h-1-m}((r-q) \tau)^{m}\right) & q<r / 2, \\
-(r-q)^{d+1} q \sum_{m=0}^{d-1}\left(\begin{array}{c}
d \\
m
\end{array}\right)\left(\bar{j}_{q}\right)_{*}\left(\left(-\widehat{\psi}_{q, q-1}\right)^{d-1-m}(q \tau)^{m}\right) & q=r / 2 .\end{cases}
$$

Therefore, for $\widehat{q}=\overline{r-q}$, we have

$$
\sum_{q=1}^{\lfloor r / 2\rfloor} \pi_{*}\left(\left(-D_{q} / r\right)^{d+1}\right)=-\frac{1}{2} \sum_{q=0}^{r-1}\left(\bar{j}_{q}\right)_{*}\left(\beta_{d-1, q}+\widehat{\beta}_{d-1, q}\right)
$$

for

$$
\begin{aligned}
& \beta_{d, q}=\frac{q \widehat{q}}{r^{d+2}} \sum_{m=0}^{d}\left(\begin{array}{c}
d+1 \\
m
\end{array}\right) \widehat{q}^{d+1} \psi_{q, q}^{d-m}(q \tau)^{m} \\
& =\frac{q \widehat{q}}{r^{d+2}}\left(\sum_{m=0}^{d+1}\left(\begin{array}{c}
d+1 \\
m
\end{array}\right) \widehat{q}^{d+1} \psi_{q, q}^{d-m}(q \tau)^{m}-\widehat{q}^{d+1} \psi_{q, q}^{-1}(q \tau)^{d+1}\right) \\
& =\frac{q \widehat{q}}{r^{d+1}} \frac{(\widehat{q} \psi)^{d+1}-((\psi+\widehat{\psi}) q \widehat{q} / r)^{d+1}}{\widehat{q} \psi-q \widehat{\psi}}
\end{aligned}
$$

and for $\widehat{\beta}_{d, q}$ defined as $\beta_{d, q}$ after the usual exchanges $q \leftrightarrow \widehat{q}$ and $\psi \leftrightarrow \widehat{\psi}$. We observe that $-((\psi+\widehat{\psi}) q \widehat{q} / r)^{d+1} /(\widehat{q} \psi-q \widehat{\psi})$ is antisymmetric with respect to such transformation. So we get

$$
\beta_{d, q}+\widehat{\beta}_{d, q}=\frac{q \widehat{q}}{r^{d+1}} \frac{(\widehat{q} \psi)^{d+1}+(q \widehat{\psi})^{d+1}}{\widehat{q} \psi-q \widehat{\psi}}=\frac{q \widehat{q}}{r^{d+1}} \sum_{a+b=d}(\widehat{q} \psi)^{a}(q \widehat{\psi})^{b} .
$$

This completes the proof of Lemma 3.1.4.

We finish the proof of the theorem by identifying the degree- $d$ term of $(20)$ with the expression (21). We have shown that the term of degree $d$ of (20) equals

$$
\frac{1}{2(d+1) !} \sum_{q=0}^{r-1}\left(\alpha_{d, q}-\beta_{d, q}-\widehat{\beta}_{d, q}+\widehat{\alpha}_{d, q}\right),
$$




\section{A. Chiodo}

with $\alpha_{d, q}$ and $\beta_{d, q}$ defined as in (27) and (31). By writing explicitly, we get

$$
\begin{aligned}
& \sum_{m=0}^{d} \frac{B_{m+2}(0)}{r^{d}}\left(\begin{array}{c}
d+2 \\
m+2
\end{array}\right) q(\widehat{q} \psi)^{d-m} \sum_{i+j=m}(r \psi)^{i}(\widehat{q} \psi-q \widehat{\psi})^{j}-\frac{q \widehat{q}}{r^{d+1}} \sum_{i+j=d}(\widehat{q} \psi)^{i}(q \widehat{\psi})^{j} \\
& \quad+\sum_{m=0}^{d} \frac{B_{m+2}(0)}{r^{d}}\left(\begin{array}{c}
d+2 \\
m+2
\end{array}\right) \widehat{q}(q \widehat{\psi})^{d-m} \sum_{i+j=m}(r \widehat{\psi})^{i}(q \widehat{\psi}-\widehat{q} \psi)^{j},
\end{aligned}
$$

which can be easily rewritten as $1 /(\psi+\widehat{\psi})$ times

$$
\begin{aligned}
& r \sum_{m=0}^{d} B_{m+2}(0)\left(\begin{array}{c}
d+2 \\
m+2
\end{array}\right)\left(\left(\frac{\widehat{q} \psi}{r}\right)^{d+1}\left(\frac{r}{\widehat{q}}\right)^{m+1}-\left(\frac{\widehat{q} \psi}{r}-\frac{q \widehat{\psi}}{r}\right)^{m+1}\left(\frac{\widehat{q} \psi}{r}\right)^{d-m}\right) \\
& \quad-q \sum_{i+j=d}\left(\frac{\widehat{q} \psi}{r}\right)^{i+1}\left(\frac{q \widehat{\psi}}{r}\right)^{j}-\widehat{q} \sum_{i+j=d}\left(\frac{\widehat{q} \psi}{r}\right)^{i}\left(\frac{q \widehat{\psi}}{r}\right)^{j+1} \\
& \quad+r \sum_{m=0}^{d} B_{m+2}(0)\left(\begin{array}{c}
d+2 \\
m+2
\end{array}\right)\left(\left(\frac{q \widehat{\psi}}{r}\right)^{d+1}\left(\frac{r}{q}\right)^{m+1}-\left(\frac{q \widehat{\psi}}{r}-\frac{\widehat{q} \psi}{r}\right)^{m+1}\left(\frac{q \widehat{\psi}}{r}\right)^{d-m}\right) .
\end{aligned}
$$

We regard the above sum as a homogeneous polynomial of degree $d+1$ in the variables $\widehat{q} \psi / r$ and $q \widehat{\psi} / r$. We show that, for any $l=1, \ldots, d$, the coefficient of the term $(\widehat{q} \psi / r)^{l}(q \widehat{\psi} / r)^{d+1-l}$ vanishes. Indeed, it equals

$$
r(-1)^{d-l} \sum_{m=d-l}^{d}\left(\begin{array}{c}
m+1 \\
d+1-l
\end{array}\right)\left(\begin{array}{c}
d+2 \\
m+2
\end{array}\right) B_{m+2}(0)-r-r(-1)^{l} \sum_{m=l-1}^{d}\left(\begin{array}{c}
m+1 \\
l
\end{array}\right)\left(\begin{array}{c}
d+2 \\
m+2
\end{array}\right) B_{m+2}(0),
$$

where the three summands correspond to the three lines in the above formula. We set $a=l$, $b=d+1-l$, and $h=m+1$, and we rewrite the above expression as

$$
-r(-1)^{b} \sum_{h=b}^{a+b}\left(\begin{array}{l}
h \\
b
\end{array}\right)\left(\begin{array}{c}
a+b+1 \\
h+1
\end{array}\right) B_{h+1}(0)-r-r(-1)^{a} \sum_{h=a}^{a+b}\left(\begin{array}{c}
h \\
a
\end{array}\right)\left(\begin{array}{c}
a+b+1 \\
h+1
\end{array}\right) B_{h+1}(0) .
$$

Note that $\{(d, l) \mid 0<l \leqslant d\}$ corresponds to $\{(a, b) \mid a, b>0\}$; therefore, we need to show that the above expression vanishes for all positive integers $a$ and $b$. The cases $a=1, b>0$ follow from (15). We can conclude by induction, because the difference between the above expression for $(a, b)$ and for $(a+1, b-1)$ equals $r$ times

$$
(-1)^{b} \sum_{h=b}^{a+b+1}\left(\begin{array}{l}
h \\
b
\end{array}\right)\left(\begin{array}{c}
a+b+1 \\
h
\end{array}\right) B_{h}(0)+(-1)^{a} \sum_{h=a+1}^{a+b+1}\left(\begin{array}{c}
h \\
a+1
\end{array}\right)\left(\begin{array}{c}
a+b+1 \\
h
\end{array}\right) B_{h}(0),
$$

which in turn equals $\left(\begin{array}{c}a+b+1 \\ b\end{array}\right)$ times

$$
(-1)^{b} \sum_{h=a+1}^{a+b+1}\left(\begin{array}{l}
a+1 \\
h-b
\end{array}\right) B_{h}(0)+(-1)^{a} \sum_{h=b}^{a+b+1}\left(\begin{array}{c}
b \\
h-a-1
\end{array}\right) B_{h}(0) .
$$

The above expression vanishes: for all nonnegative $\alpha$ and $\beta$ the $\operatorname{sum}(-1)^{\alpha} \sum_{i=0}^{\alpha}\left(\begin{array}{c}\alpha \\ i\end{array}\right) B_{i+\beta}(0)$ is equal to the sum $(-1)^{\beta} \sum_{i=0}^{\beta}\left(\begin{array}{c}\beta \\ i\end{array}\right) B_{i+\alpha}(0)$ (Carlitz identity). 


\section{ENUMERATIVE GEOMETRY OF MODULI OF $r$ th ROOTS}

In order to conclude the proof it remains to show that the coefficients of $\psi^{d+1}$ and $\widehat{\psi}^{d+1}$ equal $r B_{d+2}(\widehat{q} / r)$ and $r B_{d+2}(q / r)$, respectively. Indeed, the coefficient of $\psi^{d+1}$ is equal to

$$
\begin{aligned}
r \sum_{m=0}^{d} B_{m+2}(0)\left(\begin{array}{c}
d+2 \\
m+2
\end{array}\right)\left(\frac{\widehat{q}}{r}\right)^{d-m}-r \sum_{m=0}^{d} B_{m+2}(0)\left(\begin{array}{c}
d+2 \\
m+2
\end{array}\right)\left(\frac{\widehat{q}}{r}\right)^{d+1}-r B_{d+2}(0)\left(-\frac{\widehat{q}}{r}\right)^{d+1}-q\left(\frac{\widehat{q}}{r}\right)^{d+1} \\
=r \sum_{m=0}^{d} B_{m+2}(0)\left(\begin{array}{c}
d+2 \\
m+2
\end{array}\right)\left(\frac{\widehat{q}}{r}\right)^{d-m}-r \sum_{m=0}^{d} B_{m+2}(0)\left(\begin{array}{c}
d+2 \\
m+2
\end{array}\right)\left(\frac{\widehat{q}}{r}\right)^{d+1} \\
\quad+r B_{d+2}(1)\left(\frac{\widehat{q}}{r}\right)^{d+1}-r\left(\frac{\widehat{q}}{r}\right)^{d+1}+r\left(\frac{\widehat{q}}{r}\right)^{d+2} .
\end{aligned}
$$

Applying (15) to the three middle terms, we get

$$
r \sum_{m=0}^{d} B_{m+2}(0)\left(\begin{array}{c}
d+2 \\
m+2
\end{array}\right)\left(\frac{\widehat{q}}{r}\right)^{d-m}+r B_{1}(0)(d+2)\left(\frac{\widehat{q}}{r}\right)^{d+1}+r B_{0}(0)\left(\frac{\widehat{q}}{r}\right)^{d+2},
$$

which equals $r B_{d+2}(\widehat{q} / r)$. The same argument for the coefficient of $\psi^{d+1}$ allows us to rewrite (33) as

$$
r \frac{B_{d+2}(\widehat{q} / r) \psi^{d+1}+B_{d+2}(q / r) \widehat{\psi}^{d+1}}{\psi+\widehat{\psi}} .
$$

By $B_{n}(x)=(-1)^{n} B_{n}(1-x)$ and $-(-1)^{d+1} z^{d+1}+w^{d+1}=(z+w) \sum_{i+j=d}(-z)^{i} w^{j}$, we finally get the term (20) as desired. This completes the proof of Theorem 1.1.1.

Remark 3.1.5 (calculation of Chern classes). Let $\mathrm{ch}_{d}$ denote the term of degree $d$ of the Chern character. The $k$ th Chern class is a polynomial in the variables $\operatorname{ch}_{d}$; see $\left[\mathrm{Mac} 95, \mathrm{I} /(2.14)^{\prime}\right]$ for an explicit formula. In view of the calculation of Chern classes, in Corollary 3.1.8, we derive a formula for $\mathrm{ch}_{d}$, allowing us to express all the products of type $\mathrm{ch}_{d_{1}} \cdots \mathrm{ch}_{d_{m}}$ in terms of known products between tautological classes in the rational cohomology ring of the moduli stack of $r$-stable curves $\overline{\mathcal{M}}_{g, n}(r)$. Recall that the coarse space of $\overline{\mathcal{M}}_{g, n}(r)$ coincides with that of the Deligne-Mumford compactification $\overline{\mathcal{M}}_{g, n}$. Therefore the rational cohomology rings are isomorphic and the intersections of kappa classes, psi classes and boundary classes coincide and are known.

In order to prepare the statement of Corollary 3.1.8, we need to introduce a set of notation analogous to Remark 2.2.3.

Definition 3.1.6. Let us denote by $V$ the singular locus of the coarse universal $r$-stable curve and let us write $V^{\prime}$ for the usual double cover of $V$. A node $p \in V$ is nonseparating if the normalization at $p$ of the fibre $C \ni p$ is connected. We say that $p$ has type irr.

Let $p \in S$ be a separating node in $V$. Let $p^{\prime} \in V^{\prime}$ be a point lifting $p=\bar{\varepsilon}\left(p^{\prime}\right)$. The point $p^{\prime}$ is of type $(l, I)$, where $l \in\{0, \ldots, g\}$ and $I \subset[n]:=\{1, \ldots, n\}$ if the normalization at $p$ of the fibre $C \ni p$ is the union of two disjoint curves $C_{1}$ and $C_{2}$ containing the first and the second branches attached to $p^{\prime} \in V^{\prime}$, having genera $l$ and $g-l$, and marked at the points $\left\{x_{i} \mid i \in I\right\}$ and $\left\{x_{i} \mid i \in[n] \backslash I\right\}$, respectively.

We observe that the type is a locally constant parameter. Therefore we have

$$
V^{\prime}=V_{\text {irr }}^{\prime} \sqcup \bigsqcup_{(l, I)} V_{(l, I)}^{\prime}
$$

and the natural morphisms

$$
i_{\text {irr }}: V_{\text {irr }}^{\prime} \rightarrow \overline{\mathcal{M}}_{g, n}(r) \quad \text { and } \quad i_{(l, I)}: V_{(l, I)}^{\prime} \rightarrow \overline{\mathcal{M}}_{g, n}(r)
$$




\section{A. Chiodo}

Remark 3.1.7. We apply the above definition to the singular locus $Z$ of the universal $r$-stable curve on $\overline{\mathcal{M}}_{g, n}^{r}$ and we get

$$
Z^{\prime}=Z_{\mathrm{irr}}^{\prime} \sqcup \bigsqcup_{(l, I)} Z_{(l, I)}^{\prime} \cdot
$$

For any separating node $p$, the normalization at $p$ of the coarse space of the fibre $C \ni p$ is $C_{1} \sqcup$ $C_{2}$, where $C_{1}$ contains the first branch. Note that $p^{\prime} \in Z^{\prime}$ lifting $p$ belongs to $Z_{q}^{\prime}$ according to Notation 2.2.3(c) if and only if we have

$$
\operatorname{deg}_{C_{1}}\left(\left(\omega_{C}^{\log }\right)^{\otimes s}\left(-\sum_{i=1}^{n} m_{i}\left[x_{i}\right]\right)\right)-r \operatorname{deg}_{C_{1}}(\bar{S})=r-q,
$$

where $\bar{S}$ is the line bundle induced by the universal root via pushforward to the coarse space. It easily follows that a point $p^{\prime} \in Z^{\prime}$ of type $(l, I)$ belongs to $Z_{q}$ for $q \in\{0, \ldots, r-1\}$ satisfying

$$
q+\operatorname{deg}_{C_{1}}\left(\left(\omega_{C}^{\log }\right)^{\otimes s}\left(-\sum_{i=1}^{n} m_{i}\left[x_{i}\right]\right)\right) \in r \mathbb{Z},
$$

or equivalently

$$
q+2 l s-s-\sum_{i \in I}\left(m_{i}-s\right) \in r \mathbb{Z} .
$$

In this way we refine the decomposition of $Z^{\prime}$ given in (34), and we get the disjoint union

$$
Z^{\prime}=\bigsqcup_{q=0}^{r-1} Z_{(\mathrm{irr}, q)}^{\prime} \sqcup \bigsqcup_{(l, I)} Z_{(l, I)}^{\prime} .
$$

We also define the natural morphisms

$$
j_{(\mathrm{irr}, q)}: Z_{(\mathrm{irr}, q)}^{\prime} \rightarrow \overline{\mathcal{M}}_{g, n}(r) \quad \text { and } \quad j_{(l, I)}: Z_{(l, I)}^{\prime} \rightarrow \overline{\mathcal{M}}_{g, n}(r) .
$$

By definition $Z_{(l, I)}^{\prime}$ fits in the following fibred diagram

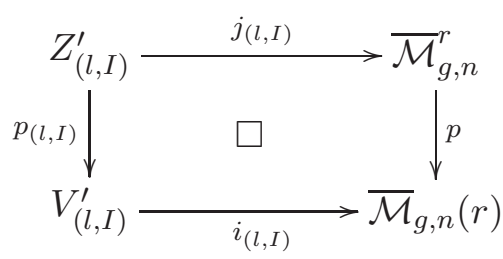

whereas, for $j_{(\mathrm{irr}, q)}$, we have the following fibred diagram.

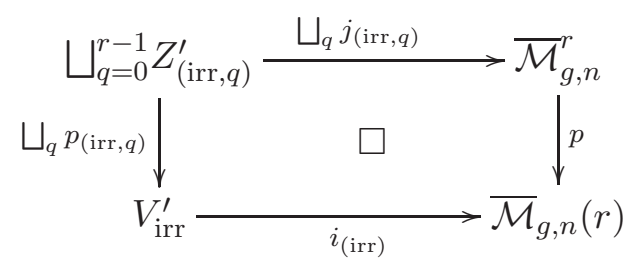

As stated in Theorem 2.2.1, we have

$$
\operatorname{deg}(p)=\operatorname{deg}\left(p_{(l, I)}\right)=r^{2 g-1},
$$

because each fibre contains $r^{2 g}$ geometric points with stabilizer $\boldsymbol{\mu}_{r}$. On the other hand, the degree of each morphism $p_{(\text {irr }, q)}$ satisfies

$$
\operatorname{deg}\left(p_{(\mathrm{irr}, q)}\right)=r^{2 g-2}
$$




\section{ENUMERATIVE GEOMETRY OF MODULI OF $r$ th ROOTS}

This means that the morphisms $p_{(\mathrm{irr}, 0)}, \ldots, p_{(\mathrm{irr}, r-1)}$ have equal degree. We show this claim by studying the fibre. Fixing a point $x^{\prime} \in V_{\text {irr }}^{\prime}$ means choosing an $n$-pointed $r$-stable curve $C$ and a nonseparating node $x \in C$, which we regard as $B \boldsymbol{\mu}_{r} \hookrightarrow C$. Furthermore, it means choosing a branch of the node, whose cotangent line can be regarded as a generator of $\operatorname{Pic}\left(B \boldsymbol{\mu}_{r}\right)$. The geometric points of the fibre of $p_{(\text {irr, }, 0)} \sqcup \cdots \sqcup p_{(\text {irr, } r-1)}$ over $x^{\prime} \in S_{\text {irr }}^{\prime}$ are all the $r^{2 g}$ distinct $r$ th roots of $\mathcal{K}$ on $C$. Each point can be assigned to a nonempty substack $H_{0}, \ldots, H_{r-1}$ according to the restriction homomorphism $\operatorname{Pic}(C) \rightarrow \operatorname{Pic}\left(B \boldsymbol{\mu}_{r}\right)$. In this way $H_{0}, \ldots, H_{r-1}$ realize the disjoint union of the fibres of $p_{(\mathrm{irr}, 0)}, \ldots, p_{(\mathrm{irr}, r-1)}$. We claim that the stacks $H_{i}$ are all isomorphic, and therefore their degree equals $(1 / r) \operatorname{deg} p$. Indeed, we can choose an $r$ th root $T_{i}$ in each substack $H_{i}$ and, via $S \mapsto S \otimes T_{i}^{\vee}$, identify $H_{i}$ to the stack of $r$-torsion line bundles that are trivially linearized at the node $x \in C$.

We denote by $\psi_{i}$ and $\kappa_{d}$ the classes defined as in Notation 3.1.1 in the cohomology of $\overline{\mathcal{M}}_{g, n}(r)$. Similarly, we define the classes $\psi$ and $\widehat{\psi}$ in $V^{\prime}$. Clearly their pullback via $p, p_{(l, I)}$, and $p_{(\text {irr }, q)}$ yields the analogue classes in $\overline{\mathcal{M}}_{g, n}^{r}$. Theorem 1.1 .1 can be written as follows.

Corollary 3.1.8. Let $\mathcal{S}$ be the universal rth root of $\left(\omega^{\log }\right)^{\otimes s}\left(-\sum_{i=1}^{n} m_{i}\left[x_{i}\right]\right)$ on the universal $r$-stable curve. We have

$$
\begin{aligned}
{\left[\operatorname{ch}\left(R^{\bullet} \pi_{*} \mathcal{S}\right)\right]_{d}=} & \left.p^{*}\left(\frac{B_{d+1}}{(d+1) !}(s / r) \kappa_{d}-\sum_{i=1}^{n} \frac{B_{d+1}}{(d+1) !}\right)\left(m_{i} / r\right) \psi_{i}^{d}+\frac{1}{2} \sum_{\substack{0 \leqslant l \leqslant g \\
I \subset[n]}} r \frac{B_{d+1}(q(l, I) / r)}{(d+1) !} i_{(l, I) *}\left(\gamma_{d-1}\right)\right) \\
& +\frac{1}{2} \sum_{q=0}^{r-1} r \frac{B_{d+1}(q / r)}{(d+1) !} j_{(\mathrm{irr}, q) *}\left(\gamma_{d-1}\right),
\end{aligned}
$$

where the cycles $\gamma_{d}$ in $A^{d}\left(V_{(l, I)}^{\prime}\right)$ and $A^{d}\left(Z_{(\mathrm{irr}, q)}^{\prime}\right)$ are defined as in Theorem 1.1.1 and $q(l, I)$ is the index fitting in (35).

\subsection{An example}

With an example we illustrate how the above formula allows us to calculate intersection numbers. When the locus $Z_{\text {irr }}^{\prime}$ of curves with nonseparating nodes is empty, the calculation can take place in the standard moduli space of stable curves. Otherwise, the above corollary and the degree evaluations (40) allow us to carry out the calculation quite easily, as the following example shows.

Example 3.2.1 (1-pointed genus-1 curves). In this example we take $s=g=n=1, m_{1}=r+1$. This means that we look at the stack $\overline{\mathcal{M}}_{1,1}^{r}$ classifying $r$ th roots of $\omega^{\log }\left(-(r+1)\left[x_{1}\right]\right)$ on $r$-stable 1-pointed genus-1 curves. In this case the direct image $R^{\bullet} \pi_{*} \mathcal{S}$ of the universal $r$ th root in the derived category is represented by $-L$ where $L$ is the line bundle $R^{1} \pi_{*} \mathcal{S}$; this happens because each fibre is irreducible and the restriction of $\mathcal{S}$ on every fibre of the universal curve $\pi: \mathcal{C} \rightarrow \overline{\mathcal{M}}_{1,1}^{r}$ has degree -1 . As we see in $\S 4.2$, this example matches Witten's predictions [Wit93] (see Example 4.2.12). We show that

$$
\operatorname{deg} \mathrm{c}_{1}\left(R^{\bullet} \pi_{*} \mathcal{S}\right)=\operatorname{deg} \mathrm{c}_{1}(-L)=-\frac{r-1}{24} .
$$

First, we use the formula of Corollary 3.1.8 for $d=1$ and get

$$
\operatorname{ch}_{1}=\frac{1}{2} p^{*}\left(B_{2}(1 / r) \kappa_{1}-B_{2}(1+1 / r) \psi_{1}\right)+\frac{1}{4} \sum_{q=0}^{r-1} r B_{2}(q / r) j_{(\mathrm{irr}, q) *}\left(\gamma_{0}\right) .
$$

Since $\pi_{*}\left(c_{1}\left(\omega_{\pi}\right)^{2}\right)$ vanishes, we have $\kappa_{1}=\psi_{1}$; we write

$$
\operatorname{ch}_{1}=\frac{1}{2}\left(B_{2}(1 / r)-B_{2}(1+1 / r)\right) p^{*} \psi_{1}+\frac{1}{4} \sum_{q=0}^{r-1} r B_{2}(q / r) j_{(\mathrm{irr}, q) *}\left(\gamma_{0}\right) .
$$




\section{A. Chiodo}

Then the relation $B_{n}(x)-B_{n}(x+1)=-n x^{n-1}$ implies that

$$
\mathrm{ch}_{1}=\frac{1}{2}(-2(1 / r)) p^{*} \psi_{1}+\frac{1}{4} \sum_{q=0}^{r-1} r\left((q / r)^{2}-(q / r)+1 / 6\right) j_{(\mathrm{irr}, q) *}\left(\gamma_{0}\right),
$$

where we used the formula for the second Bernoulli polynomial. We simplify and we evaluate $\gamma_{0}=1$,

$$
\operatorname{ch}_{1}=-p^{*} \psi_{1} / r+\frac{r}{12}\left(\bigsqcup_{q} j_{(\mathrm{irr}, q)}\right)_{*}(1 / 2)-\sum_{q=0}^{r-1} \frac{q(r-q)}{4 r} j_{(\mathrm{irr}, q) *} p_{(\operatorname{irr}, q)}^{*}(1) .
$$

We evaluate the degree via pushforward to $\overline{\mathcal{M}}_{1,1}(r)$ (recall the diagram (38)),

$$
p_{*} \operatorname{ch}_{1}=-\psi_{1}+\frac{r}{12} i_{(\mathrm{irr}) *}\left(\bigsqcup_{q} p_{(\mathrm{irr}, q)}\right)_{*}(1 / 2)-\sum_{q=0}^{r-1} \frac{q(r-q)}{4 r} i_{(\mathrm{irr}) *} p_{(\mathrm{irr}, q) *} p_{(\mathrm{irr}, q)}^{*}(1) .
$$

By (40), the degree of $p_{(\mathrm{irr}, q)}$ is 1 (one could also directly check that each fibre consists of $r$ copies of $\left.B \boldsymbol{\mu}_{r}\right)$. We have

$$
p_{*} \operatorname{ch}_{1}=-\psi_{1}+\frac{r^{2}}{12} i_{(\mathrm{irr}) *}(1 / 2)-\sum_{q=0}^{r-1} \frac{q(r-q)}{4 r} i_{(\mathrm{irr}) *}(1) .
$$

For any positive integer $r$ we have

$$
\sum_{q=1}^{r-1} q(r-q)=\frac{(r-1) r(r+1)}{6}
$$

Indeed, assume that the relation holds for $r-1$ and write $\sum_{q=1}^{r-2} q(r-q-1)=(r-2)(r-1) r / 6$. Adding $\sum_{q=1}^{r-1} q=(r-1) r / 2$ to both sides yields $\sum_{q=1}^{r-1} q(r-q)=(r-2)(r-1) r / 6+(r-1) r / 2$ and, therefore, the claim. Using the formula (45), we get

$$
p_{*} \operatorname{ch}_{1}=-\psi+\frac{r}{12} i_{(\mathrm{irr}) *}(1 / 2)-\frac{r^{2}-1}{12} i_{(\mathrm{irr}) *}(1 / 2) .
$$

Finally, since $V^{\prime}$ is a double cover of the boundary locus, we regard $i_{(\mathrm{irr}) *}(1 / 2)$ as the boundary locus of $\overline{\mathcal{M}}_{1,1}(r)$ : the fundamental class of the substack of singular $r$-stable 1-pointed genus-1 curves. Note that such a substack contains only one $r$-stable curve up to isomorphism: the 1-pointed $r$-stable curve $(X, x)$ over the nodal cubic $(\bar{X}, \bar{x})$ marked at a smooth point. Therefore, this substack is $B \operatorname{Aut}(X, x)$, where $\operatorname{Aut}(X, x)$ fits in the natural exact sequence

$$
1 \rightarrow \operatorname{Aut}((X, x),(\bar{X}, \bar{x})) \rightarrow \operatorname{Aut}(X, x) \rightarrow \operatorname{Aut}(\bar{X}, \bar{x}) \rightarrow 1,
$$

where $\operatorname{Aut}((X, x),(\bar{X}, \bar{x}))$ is the group of automorphisms fixing the coarse space and is isomorphic to $\boldsymbol{\mu}_{r}$ by [ACV03, Proposition 7.1.1]. Clearly $\operatorname{Aut}(\bar{X}, \bar{x})$ is generated by the hyperelliptic involution and has order 2. Putting everything together we get

$$
\operatorname{deg} i_{(\operatorname{irr}) *}(1 / 2)=1 / 2 r .
$$

We evaluate $\operatorname{deg} \psi=1 / 24$ (this holds for $\overline{\mathcal{M}}_{1,1}(r)$, because the morphism to $\overline{\mathcal{M}}_{1,1}$ has degree 1 and $\psi$ is a pullback from $\overline{\mathcal{M}}_{1,1}$ ). We finally reach Witten's intersection number (see $\S 4.2$ )

$$
\operatorname{deg}(-\psi)+\frac{r}{24}-\frac{r^{2}-1}{24 r}=\frac{-r+r^{2}-r^{2}+1}{24 r}=\frac{1-r}{24 r} .
$$

\subsection{A different approach via Toen's GRR formula}

As mentioned in the introduction, by a result of Toen [Toe99], the Grothendieck-Riemann-Roch calculation can be carried out entirely in the context of stacks, rather than via direct image followed 


\section{ENUMERATIVE GEOMETRY OF MODULI OF $r$ th ROOTS}

by resolution of singularities. We illustrate this in detail for the case treated in the previous example. In Remark 3.3.2, we briefly illustrate how the calculation can be carried out in general.

Example 3.3.1. Consider the moduli stack $\overline{\mathcal{M}}_{1,1}^{r}$ as in the previous example. Let $\widetilde{\pi}: \widetilde{C} \rightarrow X$ be the $r$-stable curve of genus 1 on a scheme $X$ étale on the moduli stack. Let $\Delta$ be the divisor specifying the universal marking. The universal $r$ th root is a line bundle defined on $\widetilde{C}$. Let $\widetilde{Z}$ be the singular locus of $\widetilde{C} \rightarrow X$; note that $\widetilde{Z}$ has dimension 0 and it is a union of copies of $B \boldsymbol{\mu}_{r}$. Let $\widetilde{Z}^{\prime}$ be the double cover of $\widetilde{Z}$ classifying the orders of the branches; as in Remark 2.1.5, we have

$$
\widetilde{Z}^{\prime}=\bigsqcup_{q=0}^{r-1} \widetilde{Z}_{q}^{\prime}
$$

The morphism $\tilde{\pi}$ is a morphism between a regular Deligne-Mumford stack and a regular scheme; therefore Toen's theorem applies. In order to state it, we need to introduce some constructions deriving from the initial setting.

(i) First, note that all stabilizers in $\widetilde{C}$ are isomorphic to $\boldsymbol{\mu}_{r}$. Throughout the example, $\xi$ is a primitive $r$ th root of unity.

(ii) We consider the inertia stack $I$ of $\widetilde{C}$. By definition, it is the fibre product of $\widetilde{C}$ with itself over $\widetilde{C} \times \widetilde{C}$. In this case, we have the following explicit description. We take a copy $\widetilde{C}(0)$ of $\widetilde{C}$ and $r-1$ copies $\widetilde{Z}(1), \ldots, \widetilde{Z}(r-1)$ of $\widetilde{Z}$, and we have

$$
I=\widetilde{C}(0) \sqcup \bigsqcup_{i=1}^{r-1} \widetilde{Z}(i)
$$

together with the natural morphism $\eta: I \rightarrow \widetilde{C}$. Naturally, as soon as we pass to the double cover $\widetilde{Z}^{\prime}(i)$, each copy decomposes as a disjoint union of substacks $\widetilde{Z}_{q}^{\prime}(i)$ and we have the morphisms

$$
j_{q, i}: \widetilde{Z}_{q}^{\prime}(i) \hookrightarrow \widetilde{Z}^{\prime}(i) \rightarrow I,
$$

analogous to the morphisms introduced in Notation $2.2 .3(\mathrm{~g})$. The above morphisms are defined for $0 \leqslant q<r$ and $0<i<r$; however, by taking a further copy $\widetilde{Z}(0)$ and $\widetilde{Z}^{\prime}(0)$ of $\widetilde{Z}$ and $\widetilde{Z}^{\prime}$, we extend the above definition (48) to the indices $i=0$ and $0 \leqslant q<r$ so that $j_{q, 0}$ maps to $I$ factoring through $C(0)$.

(iii) In the $K$-theory ring of $I_{\widetilde{C}}$ tensored with $\mathbb{C}$, we consider the homomorphism

$$
\rho: K^{0}(I) \otimes \mathbb{C} \rightarrow K^{0}(I) \otimes \mathbb{C}
$$

defined as follows: if a bundle $W$ is decomposed into a direct sum of eigenbundles $W^{(i)}$ with eigenvalue $\xi^{i}$,

$$
W=\bigoplus_{i=0}^{r-1} W^{(i)}, \quad \text { then we have } \quad \rho[W]=\sum_{i=0}^{r-1} \xi^{i}\left[W^{(i)}\right] .
$$

Toen's GRR formula reads

$$
\operatorname{ch}\left(R^{\bullet} \widetilde{\pi}_{*} \widetilde{S}\right)=\widetilde{\pi}_{*} \eta_{*}\left(\operatorname{ch}\left(\rho\left(\eta^{*} \widetilde{S}\right)\right) \operatorname{ch}\left(\rho\left(\lambda_{-1} \mathcal{N}_{\eta}^{\vee}\right)\right)^{-1} \operatorname{td}^{\vee}\left(\Omega_{\widetilde{\pi} \circ \eta}\right)\right)
$$

where $\lambda_{-1}$ is $\sum_{i}(-1)^{i} \bigwedge^{i}$ and we use the fact that $\operatorname{ch}\left(\rho\left(\lambda_{-1} \mathcal{N}_{\eta}^{\vee}\right)\right)$ is invertible [Toe99, Lemma 4.6].

On the connected component labelled with 0 in the inertia stack, the formula between brackets on the right-hand side yields

$$
\frac{K \exp (K / r)}{1-\exp (K)} \exp (-\Delta)+\frac{1}{12} \sum_{q=0}^{r-1}\left(j_{q, 0}\right)_{*}(1 / 2)
$$




\section{A. Chiodo}

where $K$ is the first Chern class of the relative canonical line bundle. The degree- 1 part yields the usual Riemann-Roch calculation of the degree of $R^{\bullet} \pi_{*} \mathcal{S}$. The contribution in degree 1 on $X$ comes from the classes of degree 2. After pushforward to $X$ we get

$$
-\frac{\psi_{1}}{r}+\frac{1}{12 r}\left(\bigsqcup_{q} j_{q}\right)_{*}(1 / 2)
$$

On the connected components $\widetilde{Z}_{q}^{\prime}(i)$ for $i=1, \ldots, r-1$ the formula (51) reads

$$
\frac{\xi^{q i}}{\left(1-\xi^{i}\right)\left(1-\xi^{-i}\right)}
$$

where $\xi^{q i}$ comes from the character induced by the universal $r$ th root on $\widetilde{Z}_{q}^{\prime}(i)$ and from the definition (50) of $\rho$, whereas $\left(1-\xi^{i}\right)\left(1-\xi^{-i}\right)$ is obtained after splitting $\mathcal{N}^{\vee}$ into two lines with $\boldsymbol{\mu}_{r}$-linearization $\xi \mapsto \xi$ and $\xi \mapsto \xi^{-1}$, applying the definition of $\rho$, and using the fact that $\lambda_{-1}$ is multiplicative.

A routine calculation using the formula $1 /(1-\xi)=\sum_{d=i}^{r-1}(d / r) \xi^{i}$ yields

$$
\sum_{i=1}^{r-1} \frac{\xi^{q i}}{\left(1-\xi^{i}\right)\left(1-\xi^{-i}\right)}=\frac{r^{2}-1}{12}-\frac{q(r-q)}{2} .
$$

Therefore, by pushing forward all the classes of dimension 0 mentioned above to $X$, we get

$$
-\frac{\psi_{1}}{r}+\frac{1}{12 r}\left(\bigsqcup_{q} j_{q}\right)_{*}(1 / 2)+\frac{r^{2}-1}{12 r}\left(\bigsqcup_{q} j_{q}\right)_{*}(1 / 2)-\sum_{q=1}^{r-1} \frac{q(r-q)}{2 r}\left(\bigsqcup_{q} j_{q}\right)_{*}(1 / 2) .
$$

Now, we can continue the calculation just as in the previous example, after identifying the above equation with (44).

Remark 3.3.2. We illustrate how this calculation can be carried out in general. As in the example above, the calculation consists of a first part involving the component $\widetilde{C}(0)$ in the inertia stack of $\widetilde{C}$ and a second part involving the remaining components $\widetilde{C}(1), \ldots, \widetilde{C}(r-1)$. We use the same notation as in the example.

On $\widetilde{C}(0)$, the evaluation of the product $\operatorname{ch}\left(\rho\left(\eta^{*} \widetilde{S}\right)\right) \operatorname{ch}\left(\rho\left(\lambda_{-1} \mathcal{N}_{\eta}^{\vee}\right)\right)^{-1} \operatorname{td}^{\vee}\left(\Omega_{\widetilde{\pi} \circ \eta}\right)$ appearing in Toen's formula $(51)$ can be obtained as follows. First, on $\widetilde{C}(0)$, the universal $r$ th root is a line bundle $\widetilde{S}$ and an $r$ th root of $\left(\omega^{\log }\right)^{\otimes s}\left(-\sum_{i} m_{i} \Delta_{i}\right)$. In this way, we have

$$
\operatorname{ch}(\widetilde{S})=\exp (s K / r) \prod_{i=1}^{n} \exp \left(\left(s-m_{i}\right) \Delta_{i} / r\right) .
$$

Note also that the normal sheaf $\mathcal{N}_{\eta}$ vanishes on $\widetilde{C}(0)$. The formula expressing the Todd character is the same as in Mumford's calculation. Therefore, we can proceed as in step 2 of the proof of the main theorem and we get the summands involving the kappa classes and the psi classes.

The evaluation of $\operatorname{ch}\left(\rho\left(\lambda_{-1} \mathcal{N}_{\eta}^{\vee}\right)\right)^{-1} \operatorname{td}^{\vee}\left(\Omega_{\widetilde{\pi} \circ \eta}\right)$ on the components $\widetilde{C}(1), \ldots, \widetilde{C}(r-1)$ can be found in [Tse05, $\S 7.2 .6$ ] for any twisted curve. In the case of $r$-stable curves, the expression involves cohomology classes with coefficients in the $r$ th cyclotomic field rather than $\mathbb{Q}$. Finally, $\operatorname{ch}\left(\rho\left(\eta^{*} \widetilde{S}\right)\right)$ can be evaluated as follows. Note first that the restriction of the universal $r$ th root $\widetilde{S}$ on $\widetilde{C}(1), \ldots, \widetilde{C}(r-1)$ is an $r$ th root of $\mathcal{O}$ ( $\omega$ and its twists at the markings are trivial on the singular locus). This allows us to show that, on $\widetilde{C}(i)$, the term $\operatorname{ch}\left(\rho\left(\eta^{*} \widetilde{S}\right)\right)$ can be regarded as the evaluation on $\xi^{i}$ of the character associated to $\widetilde{S}$. After these evaluations we can check that the total contribution in the cohomology ring of the base scheme yields the last summand of the formula of Theorem 1.1.1. To this effect, as in the example above, we need to express the sum of the terms with coefficients in the representations 


\section{ENUMERATIVE GEOMETRY OF MODULI OF $r$ th ROOTS}

in terms of rational coefficients. In the example, we used equation (53), whereas the general result can be obtained by applying the formula

$$
\sum_{i=1}^{r-1} \frac{\xi^{q i}}{\xi^{i} e^{x}-1}=\frac{r e^{(r-q) x}}{e^{r x}-1}-\frac{1}{e^{x}-1} .
$$

\section{Applications and motivations}

In recent years interest in the enumerative geometry of $r$ th roots has been revived by the GromovWitten theory of orbifolds and Witten's $r$-spin variant of Gromov-Witten theory. We briefly illustrate some cases where Theorem 1.1 .1 can be applied: the geometry of roots of $\mathcal{O}$ are related to the crepant resolution conjecture in the context of Gromov-Witten theory of orbifolds, whereas the roots of $\omega$ are related to Witten's $r$-spin theory.

\subsection{Enumerative geometry of the orbifold $\left[\mathbb{C}^{2} / \boldsymbol{\mu}_{r}\right]$}

As mentioned in the introduction, the crepant resolution conjecture for $\mathbb{C}^{2} / \boldsymbol{\mu}_{r}$ provides the most transparent example of an application of Theorem 1.1.1 in Gromov-Witten theory. We discuss this prototype example following [BGP08]; we only work with curves of genus 0. In view of Coates and Ruan's recent conjectures [CR07] mentioned in the introduction, one can easily adapt the algorithm illustrated here to higher genus.

As in [BGP08] we adopt a choice of a primitive $r$ th root of unity $\xi$ throughout the example. To begin with, let us recall a known fact on twisted curves (see [Cad07, Ols07]).

Remark 4.1.1 (twisted curves). We recall that the notion of twisted curves is more general than the notion of $r$-stable curves: it allows stabilizers of any finite order on the nodes and on the markings; see [AV02]. Adding stabilizers on the markings is easy: for any positive multi-index $l_{1}, \ldots, l_{n}$, there is the following equivalence of categories. On the one hand, we consider the category of $n$-pointed twisted curves with representable (scheme-theoretic) smooth locus. On the other hand, we have the category of $n$-pointed twisted curves whose $i$ th marking has stabilizer of order $l_{i}$; see $[$ Cad07, Theorem 4.1].

We show how this equivalence can be used. Let $C \rightarrow X$ be the morphism with representable smooth locus and write as $C\left(l_{1}, \ldots, l_{n}\right) \rightarrow X$ the twisted curve corresponding to $C$ in the equivalence. Then, $C\left(l_{1}, \ldots, l_{n}\right)$ carries a tautological $l_{i}$ th root $M_{i}$ of $\mathcal{O}\left(D_{i}\right)$ where $D_{i}$ is the divisor specifying the $i$ th marking. We also have a natural morphism of stacks $C\left(l_{1}, \ldots, l_{n}\right) \rightarrow C$. Any line bundle $L$ on $C\left(l_{1}, \ldots, l_{n}\right)$ can be described uniquely as a pullback from $C$ tensored with $M_{i}^{\otimes m_{i}}$ for $0 \leqslant m_{i}<l_{i}$; see [Cad07, Corollary 2.12]. In this way, any line bundle on $C\left(l_{1}, \ldots, l_{n}\right)$ induces local indices $m_{i} \in\left\{0, \ldots, l_{i}-1\right\}$ at each node. We say that $L$ is faithful if $m_{i}$ is prime to $l_{i}$. We finally recall the following fact: the pushforward via $C\left(l_{1}, \ldots, l_{n}\right) \rightarrow C$ identifies faithful $r$-torsion line bundles on the twisted curve $C\left(l_{1}, \ldots, l_{n}\right)$ with indices $m_{i}$ on the $i$ th point with $r$ th roots of $\mathcal{O}\left(-\sum_{i} r m_{i} / l_{i} D_{i}\right)$ on $C$.

We illustrate the statement. We start from the $\boldsymbol{\mu}_{r}$-action on $\mathbb{C}^{2}$,

$$
\xi \cdot(x, y) \mapsto\left(\xi x, \xi^{-1} y\right),
$$

which commutes with the action of the torus $T=\mathbb{G}_{m} \times \mathbb{G}_{m}$ on $\mathbb{C}^{2}$. The statement relates the equivariant Gromov-Witten potentials of the resolution $Y$ of the scheme $\mathbb{C}^{2} / \boldsymbol{\mu}_{r}$ and that of the stack $\mathcal{X}=\left[\mathbb{C}^{2} / \boldsymbol{\mu}_{r}\right]$. In the most recent formulation [CR07, Conjecture 10.2] the conjectural statement is that the two data can be entirely identified after a change of variables. 


\section{A. Chiodo}

The definition of the equivariant Gromov-Witten potential of $Y$ is classical; see [BGP08, $\S 2]$. Here, we illustrate how Theorem 1.1.1 applies to the calculation of the Gromov-Witten potential of $\mathcal{X}$. We set $g=0$ for simplicity. We recall the definition of the intersection numbers appearing in the equivariant genus-0 Gromov-Witten potential of $\mathcal{X}$. The $T$-action on $\mathbb{C}^{2}$ induces a $T$-action on $\mathcal{X}$. Consider the moduli stack of stable maps to $\mathcal{X}$. The inertia stack $I_{\mathcal{X}}$ has $r$ components corresponding to the elements of $\boldsymbol{\mu}_{r}$, and each component is contractible; so, the cohomology of the inertia stacks has a canonical basis $\left\{D_{0}, D_{1}, D_{2}, \ldots, D_{r-1}\right\}$, where $D_{0}$ is the class of the point at the origin in the special component of the inertia stack identified to $\mathcal{X}$. The equivariant GromovWitten potential of $\mathcal{X}$ is just a power series whose coefficients are classes in the $\operatorname{ring}$ of $T$-equivariant cohomology of the point

$$
\mathrm{GW}\left(n_{0}, \ldots, n_{r-1}\right) \in H_{T}(\mathrm{pt})=\mathbb{Q}\left[t_{1}, t_{2}\right] .
$$

This is the precise definition of $\mathrm{GW}\left(n_{0}, \ldots, n_{r-1}\right)$ in $T$-equivariant cohomology,

$$
\int_{\left.\left[\overline{\mathcal{M}}_{0, n_{0}+\cdots+n_{r-1}}(\mathcal{X}, 0)\right]\right]^{\operatorname{vir}}} \prod_{i=1}^{n_{0}} \mathrm{ev}_{i}^{*}\left(D_{0}\right) \prod_{i=n_{0}+1}^{n_{0}+n_{1}} \mathrm{ev}_{i}^{*}\left(D_{1}\right) \cdots \prod_{i=n_{0}+\cdots+n_{r-2}+1}^{n_{0}+\cdots+n_{r-1}} \mathrm{ev}_{i}^{*}\left(D_{r-1}\right),
$$

where $\overline{\mathcal{M}}_{0, n_{0}+\cdots+n_{r-1}}(\mathcal{X}, 0)$ is the stack of stable maps to $\mathcal{X}$ and ev $_{i}$ denotes the $i$ th evaluation map (see [AV02, AGV06]).

In (56), we write the formula (54) more explicitly in order to make clear that Theorem 1.1 .1 can be efficiently applied in order to calculate the invariants. We focus on the case $n_{0}=0$ and $\sum_{i} n_{i}>0$, which is the difficult part of the calculation. We proceed in three steps. In step 1, we describe more explicitly the space $\overline{\mathcal{M}}_{0, n_{0}+\cdots+n_{r-1}}(\mathcal{X}, 0)$. In step 2, we point out that the integral (54) only involves a special connected component of $\overline{\mathcal{M}}_{0, n_{0}+\cdots+n_{r-1}}(\mathcal{X}, 0)$. In step 3 , we make explicit what we mean by integration against the virtual class.

Setup. (1) the moduli stack. Since $n_{0}=0$, stable maps of degree 0 factor through $B \boldsymbol{\mu}_{r} \subset \mathcal{X}$; therefore we can integrate over $\overline{\mathcal{M}}_{0, n_{0}+\cdots+n_{r-1}}\left(B \boldsymbol{\mu}_{r}\right)$. We recall that $\overline{\mathcal{M}}_{0, n_{0}+\cdots+n_{r-1}}\left(B \boldsymbol{\mu}_{r}\right)$ is equipped with a universal curve and a universal morphism,

$$
\pi: \mathcal{C} \rightarrow \overline{\mathcal{M}}_{0, n_{0}+\cdots+n_{r-1}}\left(B \boldsymbol{\mu}_{r}\right) \quad \text { and } \quad f: \mathcal{C} \rightarrow B \boldsymbol{\mu}_{r},
$$

where $f$ is representable. The above universal object is in fact a twisted curve of genus 0 equipped with an $r$-torsion line bundle. The condition of representability imposed on $f$ can be rephrased as saying that the line bundle is faithful. Therefore, for each marking, there exist two coprime indices $(l, m)$,

$$
l \quad \text { and } \quad 0 \leqslant m<l,
$$

such that the local picture is the line bundle $L_{U}$ on $\left[U / \boldsymbol{\mu}_{l}\right]$, with $U=\operatorname{Spec} \mathbb{C}[z], \boldsymbol{\mu}_{l}$-action given by $z \mapsto \xi_{l} z$, and $L_{U}$ equal to $U \times \mathbb{C}$ with $\boldsymbol{\mu}_{l}$-linearization $\chi: \xi_{l} \mapsto \xi_{l}^{-m}$. Note that in this way we have defined the multi-indices $\left(m_{1}, \ldots, m_{n}\right)$ and $\left(l_{1}, \ldots, l_{n}\right)$, which are locally constant on $\overline{\mathcal{M}}_{0, n_{0}+\cdots+n_{r-1}}\left(B \boldsymbol{\mu}_{r}\right)$.

Setup. (2) the class $\prod_{i=1}^{n_{0}} \operatorname{ev}_{i}^{*}\left(D_{0}\right) \cdots \prod_{i=n_{0}+\cdots+n_{r-2}+1}^{n_{0}+\cdots+n_{r-1}} \operatorname{ev}_{i}^{*}\left(D_{r-1}\right)$. It is easy to see that such a class is supported on the connected component

$$
Z\left(n_{0}, n_{1}, \ldots, n_{r-1}\right) \subset \overline{\mathcal{M}}_{0, n_{0}+n_{1}+\cdots+n_{r-1}}\left(B \boldsymbol{\mu}_{r}\right)
$$

of stable maps whose local indices $\left(l_{i}, m_{i}\right)$ at the $i$ th point equal $(r / \operatorname{hcf}\{r, j\}, j / \operatorname{hcf}\{r, j\})$, where $j$ is the integer in $\{0,1, \ldots, r-1\}$ satisfying $n_{0}+\cdots+n_{j-1}+1 \leqslant i \leqslant n_{0}+\cdots+n_{j}\left(\right.$ set $\left.n_{-1}=0\right)$. 


\section{ENUMERATIVE GEOMETRY OF MODULI OF $r$ th ROOTS}

Using Remark 4.1.1, the stack $Z\left(n_{0}, n_{1}, \ldots, n_{r-1}\right)$ can be regarded as the stack of faithful $r$ th roots of

$$
\mathcal{K}=\mathcal{O}\left(-\sum_{i=n_{0}+1}^{n_{0}+n_{1}}\left[x_{i}\right]-\sum_{i=n_{0}+n_{1}+1}^{n_{0}+n_{1}+n_{2}} 2\left[x_{i}\right]-\cdots-\sum_{i=n_{0}+n_{1}+\cdots+n_{r-2}+1}^{n_{0}+n_{1}+\cdots+n_{r-1}}(r-1)\left[x_{i}\right]\right)
$$

on the universal twisted curves with trivial stabilizers at the markings.

Setup. (3) the virtual class. Finally, for dimension reasons we impose $n_{0}+n_{1}+\cdots+n_{r-1}>3$ (which amounts to requiring $n_{1}+\cdots+n_{r-1}>3$ since $n_{0}$ vanishes). Furthermore, for degree reasons we notice that the connected component $Z\left(0, n_{1}, \ldots, n_{r-1}\right)$ is empty unless we have

$$
\sum_{i} i n_{i} \in r \mathbb{Z}
$$

(this condition specializes to $n_{1} \equiv n_{2} \bmod 3$ in the case $r=3$; see [BGP08, A.2.(3)]). In the remaining cases the equivariant Gromov-Witten invariant is possibly nonzero and the virtual class can be evaluated as the Euler $T$-equivariant class of $R^{1} \pi_{*} f^{*}\left(L_{\xi} \oplus L_{\bar{\xi}}\right)$,

$$
e_{T}\left(R^{1} \pi_{*} f^{*}\left(L_{\xi} \oplus L_{\bar{\xi}}\right)\right)
$$

where $L_{\xi}$ and $L_{\bar{\xi}}$ are the line bundles on $B \boldsymbol{\mu}_{r}$ associated to the $\boldsymbol{\mu}_{r}$-representations $\xi \mapsto \xi$ and $\xi \mapsto \xi^{-1}$. Notice that $f^{*} L_{\xi}$ and $f^{*} L_{\bar{\xi}}$ on $Z\left(0, n_{1}, \ldots, n_{r-1}\right)$ can be regarded as the universal $r$ torsion line bundle $\mathcal{T}$ of $\mathcal{O}$ on $\mathcal{C}$ and its dual line bundle $\mathcal{T}^{\vee}$, respectively. For $n=\sum_{i} n_{i}>3$ and $\sum_{i} i n_{i} \in r \mathbb{Z}$, we get

$$
\mathrm{GW}\left(0, n_{1}, \ldots, n_{r-1}\right)=\operatorname{deg} e_{T}\left(R^{1} \pi_{*} f^{*}\left(L_{\xi} \oplus L_{\bar{\xi}}\right)\right)=\left(t_{1}+t_{2}\right) \operatorname{deg} \mathrm{c}_{n-3}\left(R^{1} \pi_{*}\left(\mathcal{T} \oplus \mathcal{T}^{\vee}\right)\right),
$$

where the last equality is a calculation in T-equivariant cohomology based on Mumford's proof of $c\left(\mathbb{H} \oplus \mathbb{H}^{\vee}\right)=0$; see [BGP08, Lemma 3.1].

The connection to the universal $r$ th root and to Theorem 1.1.1. The calculations (56) can be carried out on the stack $\overline{\mathcal{M}}_{0, n}^{r}$ of $r$ th roots on $n$-pointed $r$-stable curves of the line bundle $\mathcal{K}$ of $(55)$. It should be noted that the stack $\overline{\mathcal{M}}_{0, n}^{r}$ of $r$ th roots on $r$-stable curves differs from $Z\left(n_{0}, n_{1}, \ldots, n_{r-1}\right)$, because in the definition of $\overline{\mathcal{M}}_{0, n}^{r}$ we require that all stabilizers at the nodes equal $r$. However, there is a natural morphism invertible on the interior, whose ramification indices on the boundary can be explicitly handled; see [Chi08, Theorem 4.1.6].

On $\overline{\mathcal{M}}_{0, n}^{r}$, the pullback of $R^{1} \pi_{*}\left(\mathcal{T} \oplus \mathcal{T}^{\vee}\right)$ yields the direct image via the universal $r$-stable curve $\pi$ of the sum of $\mathcal{S}$ and $\mathcal{S}^{\vee}\left(-\sum_{i}\left[x_{i}\right]\right)$. Note that for both line bundles the sections of the restriction to each fibre of the $r$-stable curve $\pi$ are necessarily zero because their $r$ th power vanishes on each component (this is an easy consequence of Remark 4.1.1 or of Lemma 2.2.5 from the scheme-theoretic point of view). In this way, Theorem 1.1.1 provides the Chern character of $R^{1} \pi_{*}\left(\mathcal{T} \oplus \mathcal{T}^{\vee}\right)$, whereas the standard formulae expressing Chern classes in terms of Chern characters [Mac95, I/ $\left.(2.14)^{\prime}\right]$ allow us to derive the Gromov-Witten invariants. In general this calculation, although effective, is long to carry out in practice for high values of $n$ and $g$. For $g=0$, the formula of Corollary 3.1 .8 can be further simplified because $Z_{\text {irr }}$ is empty. In general, one can easily implement this algorithm by adapting Faber's computer program for the calculation of the intersection numbers of tautological classes.

Example 4.1.2. We calculate the genus-0 Gromov-Witten invariant corresponding to $r=5$ and

$$
\left(n_{0}, n_{1}, n_{2}, n_{3}, n_{4}\right)=(0,0,3,0,1) .
$$

This amounts to considering the moduli space of curves of genus 0 with four markings (the sum $\left.\sum_{i} n_{i}\right)$ equipped with a fifth root of $\mathcal{O}\left(-2\left[x_{1}\right]-2\left[x_{2}\right]-2\left[x_{3}\right]-4\left[x_{4}\right]\right)$. The points of this moduli functor 


\section{A. Chiodo}

can be identified with the points of the projective line $\overline{\mathcal{M}}_{0,4} \cong \mathbb{P}^{1}$, and the points of the boundary can be regarded as three points in $\mathbb{P}^{1}$. However, the automorphisms are nontrivial. Each point representing smooth curves has stabilizer $\boldsymbol{\mu}_{5}$ (the multiplication by a root of unity along the fibres of the root); furthermore, the boundary points have further automorphisms acting about the node as $(x, y) \mapsto(x, \xi y)$ (see [ACV03, Proposition 7.1.1]). In this way, this moduli functor can be regarded as a stack whose coarse space is the projective line with 25 automorphisms on three points forming the boundary locus $\Delta$ and five automorphisms on all remaining points.

In order to calculate $\operatorname{GW}(0,0,3,0,1)$, we integrate the first Chern class of the vector bundle $R^{1} \pi_{*} \mathcal{S} \oplus R^{1} \pi_{*} \mathcal{S}^{\vee}\left(-\sum_{i}\left[x_{i}\right]\right)$, where $\mathcal{S}$ is the universal root of $\mathcal{O}\left(-2\left[x_{1}\right]-2\left[x_{2}\right]-2\left[x_{3}\right]-4\left[x_{4}\right]\right)$. This amounts to evaluating the sum of the degrees of $\operatorname{ch}_{1}\left(R^{1} \pi_{*} \mathcal{S}\right)$ and $\operatorname{ch}_{1}\left(R^{1} \pi_{*} \mathcal{S}^{\vee}\left(-\sum_{i}\left[x_{i}\right]\right)\right.$. As noted above, $\mathcal{S}^{\vee}\left(-\sum_{i}\left[x_{i}\right]\right)$ can be regarded as a universal root of $\mathcal{O}\left(-3\left[x_{1}\right]-3\left[x_{2}\right]-3\left[x_{3}\right]-\left[x_{4}\right]\right)$. Then, Theorem 1.1.1 yields an identity between the terms of degree 1 of the Chern character of $R^{1} \pi_{*} \mathcal{S}$ and $R^{1} \pi_{*} \mathcal{S}^{\vee}\left(-\sum_{i}\left[x_{i}\right]\right)$ (this fact can be generalized and simplifies all calculations). Applying Corollary 3.1.8, we finally get

$$
\begin{aligned}
\operatorname{GW}(0,0,3,0,1) & =2 \operatorname{ch}_{1}\left(R^{1} \pi_{*} \mathcal{T}\right) \\
& =2\left(\frac{B_{2}(0)}{2} \kappa_{1}-\frac{B_{2}(2 / 5)}{2} \sum_{i=1}^{3} \psi_{i}-\frac{B_{2}(4 / 5)}{2} \psi_{4}+\frac{5 B_{2}(1 / 5)}{2} \Delta\right) \\
& =2\left(\frac{1}{12} \kappa_{1}+\frac{11}{300} \sum_{i=1}^{3} \psi_{i}-\frac{1}{300} \psi_{4}+\frac{5}{300} \Delta\right) \\
& =\frac{2}{5}\left(\frac{1}{12}+\frac{33}{300}-\frac{1}{300}+\frac{3}{300}\right) \\
& =\frac{2}{5}\left(\frac{1}{12}+\frac{35}{300}\right)=\frac{2}{25} .
\end{aligned}
$$

Indeed, one should notice that the Bernoulli coefficient $q(0, I)$ for all subsets of the set of markings is equal to 1 or 4 . Since $B_{2}(q / r)$ does not vary if we replace $q$ by $5-q$, all points of $\Delta$ appear with the same coefficient.

Example 4.1.3. Similarly, we get

$$
\begin{aligned}
\operatorname{GW}(0,3,1,0,0) & =2 \operatorname{ch}_{1}\left(R^{1} \pi_{*} \mathcal{T}\right) \\
& =2\left(\frac{B_{2}(0)}{2} \kappa_{1}-\frac{B_{2}(1 / 5)}{2} \sum_{i=1}^{3} \psi_{i}-\frac{B_{2}(2 / 5)}{2} \psi_{4}-\frac{5 B_{2}(2 / 5)}{2} \Delta\right) \\
& =2\left(\frac{1}{12} \kappa_{1}-\frac{1}{300} \sum_{i=1}^{3} \psi_{i}+\frac{11}{300} \psi_{4}-\frac{55}{300} \Delta\right) \\
& =\frac{2}{5}\left(\frac{1}{12}-\frac{3}{300}+\frac{11}{300}-\frac{33}{300}\right) \\
& =\frac{2}{5}\left(\frac{1}{12}-\frac{25}{300}\right)=0 .
\end{aligned}
$$

We point out that for $r=3$ the invariants $\mathrm{GW}\left(0, n_{1}, n_{2}\right)$ only depend on the sum of the entries $n_{i}$ (see [BGP08, Proposition A.1]). As we see here, this is not the case in general.

\subsection{Enumerative geometry of $r$-spin structures}

By definition $r$-spin structures are the objects of the moduli stacks $\overline{\mathcal{M}}_{g, n}^{r}$ when we set $s=1$, $2 g-2+n>0$, and $2 g-2-n-\sum_{i} m_{i} \in r \mathbb{Z}$. This section illustrates the connection between the 


\section{ENUMERATIVE GEOMETRY OF MODULI OF $r$ th ROOTS}

theory of $r$-spin curves and Theorem 1.1.1. The reader can refer to [CZ07] for a result showing the role of Theorem 1.1.1 in the more general context of Gromov-Witten theory of $r$-spin structures.

We proceed as follows: we define the $r$-spin potential $F^{r \text {-spin }}$, we provide a slight reformulation of the definition of $F^{r \text {-spin }}$ (Proposition 4.2.10), we illustrate with some examples (Examples 4.2.124.2.14) the relation with Theorem 1.1.1, and we briefly discuss further conjectural applications of Theorem 1.1.1 to the enumerative geometry of Hurwitz numbers.

The potential $F^{r \text {-spin }}$. In [Wit93] Witten introduces the potential $F^{r \text {-spin }}$, which involves the construction of $\overline{\mathcal{M}}_{g, n}^{r}$, the definition of the Witten top Chern class $\mathrm{c}_{\mathrm{W}}$, and the intersection of $\mathrm{c}_{\mathrm{W}}$ with the psi classes.

The moduli stack $\overline{\mathcal{M}}_{g, n}^{r}$ is the stack of $r$ th roots of

$$
\mathcal{K}(\vec{m})=\omega^{\log }\left(-\sum_{i} m_{i}\left[x_{i}\right]\right) .
$$

This definition makes sense for nonnegative indices $g, n$ satisfying $2 g-2+n>0$ and any multi-index $\vec{m}=\left(m_{1}, \ldots, m_{n}\right)$ satisfying $2 g-2+n-\sum_{i} m_{i} \in r \mathbb{Z}$.

The Witten top Chern class $\mathrm{c}_{\mathrm{W}}(\vec{m})$ is a cohomology class of degree $-\chi(\mathcal{S}(\vec{m}))=h^{1}(\mathcal{S}(\vec{m}))-$ $h^{0}(\mathcal{S}(\vec{m}))$ in $\overline{\mathcal{M}}_{g, n}^{r}$ (see [PV01] or [Chi06] for two different but equivalent definitions). Its definition makes sense under the above conditions on $g, n$ and $\vec{m}$ and a further condition $m_{i}>0$ for any $i$.

We define

$$
\mathrm{W}_{g, n}(\vec{a}, \vec{m})=\int_{\overline{\mathcal{M}}_{g, n}^{r}} \psi_{1}^{a_{1}} \cdots \psi_{n}^{a_{n}} \mathrm{c}_{\mathrm{W}}(\vec{m}) \in \mathbb{Q} .
$$

The definition of $F^{r \text {-spin }}$ only involves multi-indices $\vec{m}$ satisfying $0<m_{i} \leqslant r$ for any $i$.

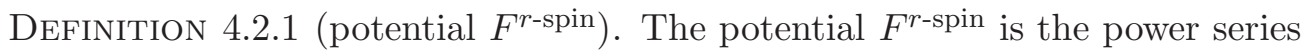

$$
F^{r \text {-spin }}=\sum_{\substack{g, n \geqslant 0 \\ 2 g-2+n>0}} \sum_{\vec{a}, \vec{m}} \frac{\mathrm{W}_{g, n}(\vec{m}, \vec{a})}{n !} \prod_{i=1}^{n} m_{i}\left(m_{i}+r\right) \cdots\left(m_{i}+r\left(a_{i}-1\right)\right)\left(m_{i}+r a_{i}\right) t_{m_{i}+r a_{i}},
$$

where $\vec{m}$ and $\vec{a}$ are multi-indices with $n$ coordinates and the summation is taken over the pairs $(\vec{a}, \vec{m})$ satisfying $0<\vec{m} \leqslant r$ and $2 g-2+n-\sum_{i} m_{i} \in r \mathbb{Z}$.

Remark 4.2.2. The integral of a cycle of positive dimension vanishes. We do not need to restrict to the multi-indices $\vec{a}$ and $\vec{m}$ for which we have $\operatorname{deg}\left(\mathrm{c}_{\mathrm{W}}(\vec{m})\right)+\sum_{i} a_{i}=\operatorname{dim} \overline{\mathcal{M}}_{g, n}^{r}$.

Remark 4.2.3. The power series $F^{r \text {-spin }}$ can be regarded as the Gromov-Witten $r$-spin potential of a point and is generalized in [JKV05] to the case where the target is a Kähler manifold.

TheOREM 4.2.4 [FSZ06] (Witten's $r$-spin conjecture [Wit93]). The power series $\exp \left(F^{r \text {-spin }}\right)$ is a tau function for the Gelfand-Dikil hierarchy $r-K d V$.

Remark 4.2.5. The statement above determines $F^{r \text {-spin }}$ and allows new calculations in the enumerative geometry of curves equipped with $r$-spin structures. Indeed, by Theorem 1.1.1, in [CZ07] we provide a tool for deriving the twisted Gromov-Witten $r$-spin potentials from $F^{r \text {-spin }}$.

Reformulation of the definition of $F^{r \text {-spin }}$. Using a result of Polishchuk and Vaintrob (see Lemma 4.2.8) conjectured by Jarvis, Kimura, and Vaintrob [JKV01b], we reformulate the above definition of $F^{r \text {-spin }}$ via a change of variables. (This change of variables is also suggested in [JKV01b, Remark 2.3].) 


\section{A. Chiodo}

Recall Remark 4.2.2: either we can sum over all indices $\vec{m}$ and $\vec{a}$ for which $d$ divides the degree $2 g-2+n-\sum_{i} m_{i} \in r$, or we can select those multi-indices $\vec{m}$ and $\vec{a}$ for which the dimension of $\mathcal{M}_{g, n}^{r}$ is equal to $\operatorname{deg}\left(\mathrm{c}_{\mathrm{W}}(\vec{m})\right)+\sum_{i} a_{i}$. It turns out that this second choice has several advantages. Let us make this condition explicit.

It is easy to see that $\vec{m}$ and $\vec{a}$ satisfy $\operatorname{deg}\left(\mathrm{c}_{\mathrm{W}}(\vec{m})\right)+\sum_{i} a_{i}=\operatorname{dim} \overline{\mathcal{M}}_{g, n}^{r}$ if and only if

$$
\sum_{i}\left(r a_{i}+m_{i}\right)=(2 g-2+n)(r+1) .
$$

Remark 4.2.6. An immediate advantage of imposing (57) directly in the definition of $F^{r \text {-spin is that }}$ we do not need to impose the condition $2 g-2+n-\sum_{i} m_{i} \in r \mathbb{Z}$ : it follows as an immediate consequence.

Remark 4.2.7. A less obvious advantage of imposing (57) is that each number $\mathrm{W}(\vec{a}, \vec{m})$ can be computed without involving psi classes. We see how in two steps.

First, we change parameters. Equation (57) suggests that the two multi-indices $\vec{m}$ and $\vec{a}$ can be incorporated in a single multi-index

$$
\vec{k}=r \vec{a}+\vec{m} .
$$

Instead of starting from two multi-indices $\vec{m}$ and $\vec{a}$ satisfying (57), we can start from a single multi-index $\vec{k}$ satisfying

$$
\sum_{i} k_{i}=(2 g-2+n)(r+1) .
$$

The datum of a positive multi-index $\vec{k}$ and that of two multi-indices $\vec{m} \in\{1, \ldots, r\}^{n}$ and $\vec{a} \in\left(\mathbb{Z}_{\geqslant 0}\right)^{n}$ are interchangeable.

Second, we take $\vec{k}=r \vec{a}+\vec{m}$ and we calculate $\mathrm{W}_{g, n}(\vec{m}, \vec{a})$ without psi classes. On the one hand, recall that by definition $\mathrm{W}_{g, n}(\vec{m}, \vec{a})$ is the cup product $\psi_{1}^{a_{1}} \cdots \psi_{n}^{a_{n}} \mathrm{cW}(\vec{m})$. On the other hand, compute $\mathrm{W}_{g, n}(\vec{k}, \overrightarrow{0})$; explicitly, this means take the moduli stack of $r$ th $\operatorname{roots}$ of $\mathcal{K}(\vec{k})$ and apply the construction of $\mathrm{c}_{\mathrm{W}}$ to the universal $r$ th root $\mathcal{S}(\vec{k})$ (as pointed out above this construction makes sense as soon as the multi-index is positive and not only inside $\{1, \ldots, r\}$ ). Since $\vec{k}$ satisfies $(58)$ we get a cycle $\mathrm{c}_{\mathrm{W}}(\vec{k})$ of dimension 0 and therefore, by integrating, a number. The following lemma shows how $\mathrm{W}_{g, n}(\vec{m}, \vec{a})$ can be computed from $\mathrm{W}_{g, n}(\vec{k}, \overrightarrow{0})$.

Lemma 4.2.8 [PV01, Proposition 5.1]. The classes $\mathrm{c}_{\mathrm{W}}(\vec{m})$ and $\mathrm{c}_{\mathrm{W}}(\vec{k})=\mathrm{cW}_{\mathrm{W}}(r \vec{a}+\vec{m})$ satisfy

$$
\mathrm{c}_{\mathrm{W}}(\vec{k})=\left(\prod_{i=1}^{n} \frac{m_{i} \cdots\left(m_{i}+\left(a_{i}-1\right) r\right)}{r^{a_{i}}}\right) \psi_{i}^{a_{1}} \cdots \psi_{n}^{a_{n}} \mathrm{c}_{\mathrm{W}}(\vec{m}) .
$$

Corollary 4.2.9. For $\vec{m}$ and $\vec{a}$ satisfying (57) and $0<m_{i} \leqslant r$, we have

$$
\mathrm{W}_{g, n}(\vec{m}, \vec{a})=\left(\prod_{i=1}^{n} \frac{r^{a_{i}}}{m_{i} \cdots\left(m_{i}+\left(a_{i}-1\right) r\right)}\right) \mathrm{W}_{g, n}(0, \vec{k}),
$$

where $\vec{k}$ equals $r \vec{a}+\vec{m}$.

We get the following simplified definition of the potential $F^{r \text {-spin }}$.

Proposition 4.2.10. We assume as usual $r \geqslant 2$ and $g$, $n$ satisfying $(2 g-2+n)>0$. We have

$$
F^{r \text {-spin }}=\sum_{\substack{g, n \geqslant 0 \\ g-2+n>0}} \sum_{\substack{k_{i}>0 \\ \sum_{i} k_{i}=(2 g-2+n)(r+1)}} \frac{\operatorname{deg} \mathrm{c}_{\mathrm{W}}(\vec{k})}{n !} \prod_{i=1}^{n} m_{i} r^{\left\lfloor m_{i} / r\right\rfloor} t_{m_{i}},
$$

where $\mathrm{c}_{\mathrm{W}}(\vec{k})$ is Witten's top Chern associated to the universal $r$ th root of $\omega^{\log }\left(-\sum_{i} k_{i}\left[x_{i}\right]\right)$. 


\section{ENUMERATIVE GEOMETRY OF MODULI OF $r$ th ROOTS}

The Witten top Chern class and the direct image $R^{\bullet} \pi_{*} \mathcal{S}(\vec{k})$. We recall from [PV01] and [Chi06] that the class $\mathrm{c}_{\mathrm{W}}(\vec{k})$ is determined by the direct image $R^{\bullet} \pi_{*} \mathcal{S}(\vec{k})$ and by a natural homomorphism in the derived category induced by $\mathcal{S}(\vec{k})^{\otimes r} \cong \mathcal{K}(\vec{k})$ and Serre duality:

$$
\operatorname{Sym}^{r}\left(R^{\bullet} \pi_{*} \mathcal{S}\right) \rightarrow \mathcal{O}[-1] .
$$

Such a class is a generalization of the top Chern class: it coincides with $c_{\text {top }}\left(R^{1} \pi_{*} \mathcal{S}\right)$ when $\mathcal{S}$ has no sections along each fibre of the universal $r$-stable curve (note that this implies that $\pi_{*} \mathcal{S}=0$ and $R^{1} \pi_{*} \mathcal{S} \in$ Vect). In general $\mathrm{c}_{\mathrm{W}}$ is not the top Chern class of a vector bundle, but it has the same cohomological degree as $-\chi(\mathcal{S}(\vec{k}))$.

Remark 4.2.11. We point out that for $\vec{k}$ satisfying $\sum_{i=1}^{n} k_{i}=(r+1)(2 g-2+n)$ we have $-\chi(\mathcal{S})=$ $\operatorname{dim}\left(\overline{\mathcal{M}}_{g, n}^{r}\right)$. Furthermore $\sum_{i} k_{i}=(r+1)(2 g-2+n)$ implies that the direct image $\pi_{*} L$ vanishes, because the relative degree of the universal $r$ th root of $\mathcal{K}(\vec{k})$ is negative:

$$
\operatorname{deg}_{\pi} \mathcal{S}(\vec{k})=\frac{2 g-2+n-\sum_{i=1}^{n} m_{i}}{r}=-(2 g-2+n)<0 .
$$

On the open substack of $r$ th roots defined on irreducible $r$-stable curves, the direct image $R^{1} \pi_{*} L$ is a vector bundle of $\operatorname{rank}-\chi(\mathcal{S})=\operatorname{dim}\left(\overline{\mathcal{M}}_{g, n}^{r}\right)$, because the $r$-spin structure has no section on each fibre. Away from the locus classifying irreducible curves, the direct image is not a vector bundle in general. We show this with some examples.

Assuming $\sum_{i} k_{i}=(r+1)(2 g-2+n)$, we provide three examples. In the first case, $R^{1} \pi_{*} \mathcal{S}$ is locally free and Witten's top Chern class is its top Chern class. In the second and third examples, $R^{1} \pi_{*} \mathcal{S}$ has torsion (even if the condition $\sum_{i} k_{i}=(r+1)(2 g-2+n)$ guarantees that $\pi_{*} \mathcal{S}$ vanishes, the sheaf $R^{1} \pi_{*} \mathcal{S}$ is not locally free). The second example shows that this happens in codimension 1 inside any moduli space $\overline{\mathcal{M}}_{g, n}^{r}$ for $g>0$ and $(g, n) \neq(1,1)$. The third example has the advantage of being very elementary and concrete, stack-theoretic curves do not appear.

Example 4.2.12. Since every 1-pointed 1-stable curve is irreducible, we deduce that $\operatorname{deg} \mathrm{c}_{\mathrm{W}}$ $(r+1)$ can be regarded as the first Chern class of the line bundle $R^{1} \pi_{*} \mathcal{S}$ on the stack $\overline{\mathcal{M}}_{1,1}^{r}$ of $r$ th roots of $\omega_{\pi}\left(-r\left[x_{1}\right]\right)$. In this way, using the calculation carried out in Example 3.2.1 and the above Lemma 4.2.8, we get a new verification of the prediction of Witten [Wit93],

$$
\int_{\overline{\mathcal{M}}_{1,1}^{r}} \psi_{1} \mathrm{c}_{\mathrm{W}}(1)=\mathrm{W}_{1,1}(1,1)=r \mathrm{~W}_{1,1}(0, r+1)=\frac{r-1}{24} .
$$

See [JKV01a, 4.4], [PV01, 5.3/3], and [Chi06, 6.4] for different verifications of the same invariant.

Example 4.2.13. We exhibit an $r$-spin structure on a curve with $n$ markings and multi-index $\vec{k}$ satisfying $\sum_{i} k_{i}=(r+1)(2 g-2+n)$. Consider a curve $C$ of genus $g$ with one node and two smooth irreducible components of genus 1 and $g-1$. Assume that the markings belong to the component of genus $g-1$. Note that, for degree reasons, all $r$-spin structures are defined on the curve having stabilizer of order $r$ on the node. Furthermore, since all markings are on the component of genus $g-1$, the sections of any $r$-spin structure constantly vanish on the component of genus $g-1$.

We are interested in the group of sections $H^{0}$ of these $r$-spin structures. We want to show that, even if $\sum_{i} k_{i}=(r+1)(2 g-2+n)$ is satisfied, it may well happen that $H^{0}$ does not vanish. Using Lemma 2.2.5, one can regard the sections of any $r$-spin structure on $C$ as sections of a line bundle $S^{\prime}$ on a nodal curve $C^{\prime}$ obtained from $C$ by normalization and by gluing a projective line to the two preimages of the node. There exists an $r$-spin structure for which the corresponding line bundle $S^{\prime}$ on $C^{\prime}$ is simply obtained by gluing three line bundles defined on each component and verifying the following conditions: the line bundle on the component of genus 1 is trivial, the line bundle on the rational component is isomorphic to $\mathcal{O}(1)$, and the line on the remaining component has only one global section $s=0$. Now it is immediate to see that for such a line bundle $H^{0}$ is one-dimensional. 


\section{A. Chiodo}

Example 4.2.14. Set $r=2$, consider a curve of genus $g>1$ with an even number $n$ of markings, and choose a multi-index $k_{1}, \ldots, k_{n}$ such that $\sum_{i} k_{i}=3(2 g-2+n)$. If the curve is smooth, the 2 -spin structures are square roots of a line bundle of degree $-2(2 g-2+n)$, therefore for all 2-spin structures we have $H^{0}=0$. We let the curve degenerate and acquire two nonseparating nodes: we now look at a curve $C$ that is the union of a projective line $P$ and a curve $E$ of genus $g-1$ meeting at two points. We choose the nodal curve in such a way that the markings belong to the irreducible component of genus 0 (in this way the nodal curve is stable). We consider a square root of $\omega_{C}^{\log }\left(-\sum k_{i}\left[x_{i}\right]\right)$. Its degree on $P$ is $-\sum_{i}\left(k_{i}-1\right) / 2=-3(g-1)-n$, whereas on $E$ the degree is $g-1$. Since the degree on $P$ is strictly negative, the sections of a square root $S$ in $\operatorname{Pic} C$ are sections of the restriction on $E$ vanishing at the points meeting $P$. By construction, the restriction $\left.S\right|_{E}$ is a theta characteristic of the curve $E$ of genus $g-1$. We can easily choose from the start a curve $E$ with a theta characteristic having more than two linearly independent sections (see [Mum71, §4] for explicit examples).

The problem of finding an explicit closed formula for Witten's $r$-spin invariants is still open. Using the cohomological field theory axioms [JKV01a], Shadrin and Zvonkine [SZ08] provide an algorithm. More generally, it would be interesting to know how Witten's top Chern class is related to the tautological classes (see [FJR07, Conjecture 2.5.3]) and to the Chern classes of $R^{\bullet} \pi_{*} \mathcal{S}$ (see the discussion in $[\mathrm{Pol04}])$.

The Chern classes of $R^{\bullet} \pi_{*} \mathcal{S}$ and Hurwitz numbers. Recently, pursuing a different approach to Witten's statement, Zvonkine formulated new conjectures on the enumerative geometry of the $r$-spin structures. He starts from the Ekedahl-Lando-Shapiro-Vainshtein formula [ELSV01]

$$
h_{g ; b_{1}, \ldots, b_{n}}=(d+n+2 g-2) ! \prod_{i=1}^{n} \frac{b_{i}^{b_{i}}}{b_{i} !} \int_{\overline{\mathcal{M}}_{g, n}} \frac{1-\lambda_{1}+\lambda_{2}-\cdots \pm \lambda_{g}}{\left(1-b_{1} \psi_{1}\right) \cdots\left(1-b_{n} \psi_{n}\right)},
$$

where $d=\sum_{i} b_{i}, \lambda_{k}$ denotes the $k$ th Chern class of the Hodge bundle, and $h_{g ; b_{1}, \ldots, b_{n}}$ is the Hurwitz number of simply ramified covers of the sphere with profile $\left(b_{1}, \ldots, b_{n}\right)$ at infinity. His conjectural formula involves in a similar way the Chern classes $\mathrm{c}_{k}\left(R^{\bullet} \pi_{*} \mathcal{S}\right)$ (see [Zvo08]).

\section{ACKNOWLEDGEMENTS}

I would like to thank D. Zvonkine for pointing out Example 4.2.13 to me and for showing me his conjectural statement relating $r$-spin curves and Hurwitz numbers. In the course of the proof of Theorem 1.1.1, we handle the degeneration of roots by means of $q$-chain divisors. These divisors are implicitly used by L. Caporaso, C. Casagrande, and M. Cornalba in [CCC07], and I am grateful to M. Cornalba for illustrating them to me (see Figure 3). I would like to thank J. Bertin and the anonymous referee for the care with which they read this work and for their precious comments and corrections. My thanks to S. Boissière, N. Borne, T. Graber, F. Herbaut, A. Hirschowitz, T. Rivoal, and C. Simpson for helpful conversations and advice.

\section{REFERENCES}

ACV03 D. Abramovich, A. Corti and A. Vistoli, Twisted bundles and admissible covers, Comm. Algebra 31 (2003), 3547-3618.

AJ03 D. Abramovich and T. J. Jarvis, Moduli of twisted spin curves, Proc. Amer. Math. Soc. 131 (2003), 685-699.

AGV06 D.Abramovich, D. Graber and A. Vistoli, Gromov-Witten theory of Deligne-Mumford stacks, Preprint (2006), math.AG/0603151 


\section{ENUMERATIVE GEOMETRY OF MODULI OF $r$ th ROOTS}

AV02 D. Abramovich and A. Vistoli, Compactifying the space of stable maps, J. Amer. Math. Soc. 15 (2002), 27-75.

AC87 E. Arbarello and M. Cornalba, The Picard groups of the moduli spaces of curves, Topology 26 (1987), 153-171.

BG06 J. Bryan and T. Graber, The crepant resolution conjecture, Algebraic Geometry, Seattle, 2005, to appear; Preprint (2006), math.AG/0610129.

BGP08 J. Bryan, T. Graber and R. Pandharipande, The orbifold quantum cohomology of $\mathbb{C}^{2} / \mathbb{Z}_{3}$ and Hurwitz-Hodge integrals, J. Algebraic Geom. 17 (2008), 1-28.

Cad07 C. Cadman, Using stacks to impose tangency conditions on curves, Amer. J. Math. 129 (2007), 405-427.

CCC07 L. Caporaso, C. Casagrande and M. Cornalba, Moduli of roots of line bundles on curves, Trans. Amer. Math. Soc. 359 (2007), 3733-3768.

Chi06 A. Chiodo, The Witten top Chern class via K-theory, J. Algebraic Geom. 15 (2006), 681-707.

Chi08 A. Chiodo, Stable twisted curves and their r-spin structures, Ann. Inst. Fourier (Grenoble) $\mathbf{5 8}$ (2008), 1635-1689.

CCIT07 T. Coates, A. Corti, H. Iritani and H.-H. Tseng, The crepant resolution conjecture for type A surface singularities, Preprint (2007), arXiv:0704.2034.

CR07 T. Coates and Y. Ruan, Quantum cohomology and crepant resolutions: A conjecture. Preprint (2007), arXiv:0710.5901.

Cor89 M. Cornalba, Moduli of curves and theta-characteristics, in Lectures on Riemann surfaces, eds M. Cornalba, X. Gomez-Mont and A. Verjovsky (World Scientific, Singapore, 1989), 560-589.

CZ07 A. Chiodo and D. Zvonkine. Twisted Gromov-Witten r-spin potentials and Givental's quantization, Preprint (2007), arXiv:0711.0339.

DM69 P. Deligne and D. Mumford, Irreducibility of the space of curves of given genus, Publ. Math. Inst. Hautes Études Sci. 36 (1969), 75-112.

ELSV01 T. Ekedahl, S. Lando, M. Shapiro and A. Vainshtein, Hurwitz numbers and intersections on moduli spaces of curves, Invent. Math. 146 (2001), 297-372.

FSZ06 C. Faber, S. Shadrin and D. Zvonkine, Tautological relations and the r-spin Witten conjecture, Preprint (2006), math.AG/0612510.

Fal96 G. Faltings, Moduli-stacks for bundles on semistable curves, Math. Ann. 304 (1996), 489-515.

FJR07 H. Fan, T. Jarvis and Y. Ruan, The Witten equation, mirror symmetry and quantum singularity theory, Preprint (2007), arXiv:0712.4021v1

Jar98 T. J. Jarvis, Torsion-free sheaves and moduli of generalized spin curves, Compositio Math. 110 (1998), 291-333.

Jar00 T. J. Jarvis, Geometry of the moduli of higher spin curves, Internat. J. Math. 11 (2000), 637-663.

Jar01 T. J. Jarvis, The Picard group of the moduli of higher spin curves, New York J. Math. 7 (2001), $23-47$.

JKV01a T. J. Jarvis, T. Kimura and A. Vaintrob, Moduli spaces of higher spin curves and integrable hierarchies, Compositio Math. 126 (2001), 157-212.

JKV01b T. J. Jarvis, T. Kimura and A. Vaintrob, Gravitational descendants and the moduli space of higher spin curves, in Advances in algebraic geometry motivated by physics, Lowell, MA, 2000, ed. E. Previato, Contemporary Mathematics, vol. 276 (American Mathematical Society, Providence, RI, 2001) 167-177.

JKV05 T. J. Jarvis, T. Kimura and A. Vaintrob, Stable spin maps, Gromov-Witten invariants, and quantum cohomology, Comm. Math. Phys. 259 (2005), 511-543.

Kon92 M. Kontsevich, Intersection theory on the moduli space of curves and the matrix Airy function, Comm. Math. Phys. 147 (1992), 1-23.

LM00 G. Laumon and L. Moret-Bailly, Champs algébriques (Springer, Berlin, 2000).

Mac95 I. G. Macdonald, Symmetric functions and Hall polynomials, second edition (Clarendon Press, Oxford, 1995). 


\section{ENUMERATIVE GEOMETRY OF MODULI OF $r$ th ROOTS}

Mum71 D. Mumford, Theta characteristics on an algebraic curve, Ann. Sci. École Norm. Sup. (4) (1971), 181-192.

Mum83 D. Mumford, Towards an enumerative geometry of the moduli space of curves in Arithmetic and geometry, vol. II, Progress in Mathematics, vol. 36 (Birkhäuser, Boston, MA, 1983), 271-328.

Ols07 M. Olsson, On (log) twisted curves, Compositio Math. 143 (2007), 476-494.

PV01 A. Polishchuk and A. Vaintrob, Algebraic construction of Witten's top Chern class, in Advances in algebraic geometry motivated by physics, Lowell, MA, 2000, ed. E. Previato, Contemporary Mathematics, vol. 276 (American Mathematical Society, Providence, RI, 2001), 229-250,

Pol04 A. Polishchuk, Witten's top Chern class on the moduli space of higher spin curves, in Frobenius manifolds, Aspects of Mathematics, vol. 36 (Vieweg, Wiesbaden, 2004), 253-264.

Tse05 H.-H. Tseng, Orbifold quantum Riemann-Roch, Lefschetz and Serre, Preprint (2005), math.AG/0506111.

Toe99 B. Toen, Théorèmes de Riemann-Roch pour les champs de Deligne-Mumford, K-Theory 18 (1999), 33-76.

Wit93 E. Witten, Algebraic geometry associated with matrix models of two-dimensional gravity, in Topological methods in modern mathematics, Stony Brook, NY, 1991 (Publish or Perish, Houston, TX, 1993), 235-269.

SZ08 S. Shadrin and D. Zvonkine, Intersection numbers with Witten's top Chern class, Geom. Topol. 12 (2008), 713-745.

Zvo08 D. Zvonkine, Intersection theory on moduli spaces and integrable hierarchies, at Géométrie algébrique et géométrie complexe 2008, http://www.cirm.univ-mrs.fr/

Alessandro Chiodo chiodo@ujf-grenoble.fr

Institut Fourier, UMR CNRS 5582, UFR de Mathématiques, Université de Grenoble 1, BP 74, 38402 Saint Martin d'Hères, France 